\title{
Two Curves, One Price: Pricing 83 Hedging Interest Rate Derivatives Decoupling Forwarding and Discounting Yield Curves
}

\author{
Marco Bianchetti *
}

\author{
Risk Management, Market Risk, Pricing and Financial Modeling, \\ Intesa Sanpaolo, piazza P. Ferrari 10, 20121 Milan, Italy, \\ e-mail: marco.bianchetti(at)intesasanpaolo.com
}

First version: 14 Nov. 2008, this version: 1 Aug. 2012.

A shorter version of this paper is published in Risk Magazine, Aug. 2010.

\begin{abstract}
We revisit the problem of pricing and hedging plain vanilla single-currency interest rate derivatives using multiple distinct yield curves for market coherent estimation of discount factors and forward rates with different underlying rate tenors.

Within such double-curve-single-currency framework, adopted by the market after the credit-crunch crisis started in summer 2007, standard single-curve noarbitrage relations are no longer valid, and can be recovered by taking properly into account the forward basis bootstrapped from market basis swaps. Numerical results show that the resulting forward basis curves may display a richer microterm structure that may induce appreciable effects on the price of interest rate instruments.

By recurring to the foreign-currency analogy we also derive generalised noarbitrage double-curve market-like formulas for basic plain vanilla interest rate derivatives, FRAs, swaps, caps/floors and swaptions in particular. These expressions include a quanto adjustment typical of cross-currency derivatives, naturally originated by the change between the numeraires associated to the two yield curves, that carries on a volatility and correlation dependence. Numerical scenarios confirm that such correction can be non negligible, thus making unadjusted double-curve prices, in principle, not arbitrage free.

Both the forward basis and the quanto adjustment find a natural financial explanation in terms of counterparty risk.

${ }^{*}$ The author acknowledges fruitful discussions with M. Blatter, M. De Prato, M. Henrard, M. Joshi, C. Maffi, G. V. Mauri, F. Mercurio, N. Moreni, A. Pallavicini, many colleagues in the Risk Management and participants at Quant Congress Europe 2009. A particular mention goes to M. Morini and M. Pucci for their encouragement, and to F. M. Ametrano and the QuantLib community for the open-source developments used here. The views expressed here are those of the author and do not represent the opinions of his employer. They are not responsible for any use that may be made of these contents.
\end{abstract}


JEL Classifications: E43, G12, G13.

Keywords: liquidity, crisis, counterparty risk, yield curve, forward curve, discount curve, pricing, hedging, interest rate derivatives, FRAs, swaps, basis swaps, caps, floors, swaptions, basis adjustment, quanto adjustment, measure changes, no arbitrage, QuantLib.

\section{Contents}

1 Introduction

2 Notation and Basic Assumptions

3 Pre and Post Credit Crunch Market Practices 6

3.1 Single-Curve Framework . . . . . . . . . . . . . . . . 6

3.2 Multiple-Curve Framework . . . . . . . . . . . . . . . . 8

4 No Arbitrage and Forward Basis $\quad 10$

5 Foreign-Currency Analogy and Quanto Adjustment 14

5.1 Forward Rates . . . . . . . . . . . . . . . . . 15

5.2 Swap Rates . . . . . . . . . . . . . . . . . . . . . 18

6 Double-Curve Pricing \& Hedging Interest Rate Derivatives 20

6.1 Pricing . . . . . . . . . . . . . . . . . . . . . . . . . . . . . . . . . . .

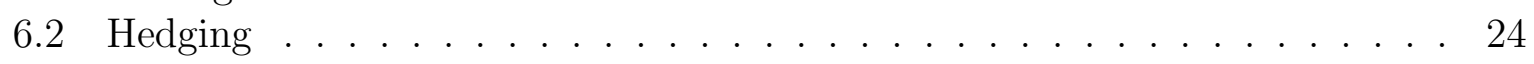

7 No Arbitrage and Counterparty Risk 25

8 Conclusions $\quad 26$ 


\section{Introduction}

The credit crunch crisis started in the second half of 2007 has triggered, among many consequences, the explosion of the basis spreads quoted on the market between single-currency interest rate instruments, swaps in particular, characterised by different underlying rate tenors (e.g. Xibor3M 11, Xibor6M, etc.). In fig. 1 we show a snapshot of the market quotations as of Feb. 16th, 2009 for the six basis swap term structures corresponding to the four Euribor tenors 1M, 3M, 6M, 12M. As one can see, in the time interval $1 Y-30 Y$ the basis spreads are monotonically decreasing from 80 to around 2 basis points. Such very high basis reflect the higher liquidity risk suffered by financial institutions and the corresponding preference for receiving payments with higher frequency (quarterly instead of semi-annually, etc.).

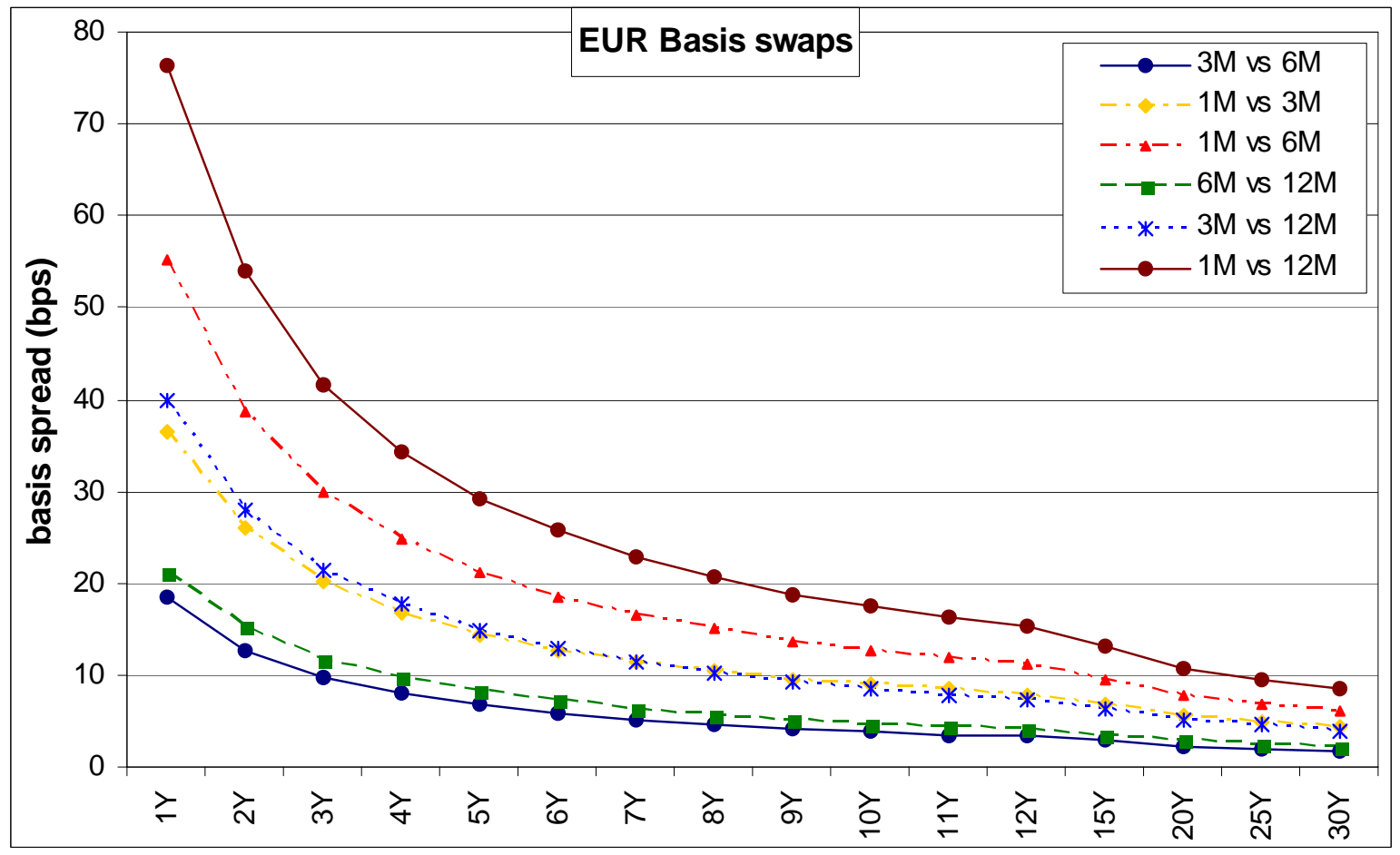

Figure 1: quotations (basis points) as of Feb. 16th, 2009 for the six EUR basis swap curves corresponding to the four Euribor swap curves 1M, 3M, 6M, 12M. Before the credit crunch of Aug. 2007 the basis spreads were just a few basis points (source: Reuters page ICAPEUROBASIS).

There are also other indicators of regime changes in the interest rate markets, such as the divergence between deposit (Xibor based) and OIS 2 (Eonia 3 based for EUR) rates,

\footnotetext{
${ }^{1}$ We denote with Xibor a generic Interbank Offered Rate. In the EUR case the Euribor is defined as the rate at which euro interbank term deposits within the euro zone are offered by one prime bank to another prime bank (see www.euribor.org).

${ }^{2}$ Overnight Indexed Swaps.

${ }^{3}$ Euro OverNight Index Average, the rate computed as a weighted average of all overnight rates corresponding to unsecured lending transactions in the euro-zone interbank market (see e.g. www.euribor.org).
} 
or between FRA4 contracts and the corresponding forward rates implied by consecutive deposits (see e.g. refs. AB09, Mer09, [Mor08, [Mor09]).

These frictions have thus induced a sort of "segmentation" of the interest rate market into sub-areas, mainly corresponding to instruments with 1M, 3M, 6M, 12M underlying rate tenors, characterized, in principle, by different internal dynamics, liquidity and credit risk premia, reflecting the different views and interests of the market players. We stress that market segmentation was already present (and well understood) before the credit crunch (see e.g. ref. [TP03]), but not effective due to negligible basis spreads.

Such evolution of the financial markets has triggered a general reflection about the methodology used to price and hedge interest rate derivatives, namely those financial instruments whose price depends on the present value of future interest rate-linked cash flows. In this paper we acknowledge the current market practice, assuming the existence of a given methodology (discussed in detail in ref. [AB09]) for bootstrapping multiple homogeneous forwarding and discounting curves, characterized by different underlying rate tenors, and we focus on the consequences for pricing and hedging interest rate derivatives. In particular in sec. 3 we summarise the pre- and post-credit crunch market practices for pricing and hedging interest rate derivatives. In sec. 2 we fix the notation, we revisit some general concept of standard, no arbitrage single-curve pricing and we formalize the double-curve pricing framework, showing how no arbitrage is broken and can be formally recovered with the introduction of a forward basis. In sec. 5 we use the foreign-currency analogy to derive a single-currency version of the quanto adjustment, typical of cross-currency derivatives, naturally appearing in the expectation of forward rates. In sec. 6 we derive the no arbitrage double-curve market-like pricing expressions for basic single-currency interest rate derivatives, such as FRA, swaps, caps/floors and swaptions. Conclusions are summarised in sec. 8 ,

The topic discussed here is a central problem in the interest rate market, with many consequences in trading, financial control, risk management and IT, which still lacks of attention in the financial literature. To our knowledge, similar topics have been approached in refs. [FZW95], [BS05], [KTW08], [Mer09], [Hen09] and [Mor08], Mor09] . In particular W. Boenkost and W. Schmidt BS05] discuss two methodologies for pricing cross-currency basis swaps, the first of which (the actual pre-crisis common market practice), does coincide, once reduced to the single-currency case, with the double-curve pricing procedure described her5. Recently M. Kijima et al. KTW08 have extended the approach of ref. [BS05] to the (cross currency) case of three curves for discount rates, Libor rates and bond rates. Finally, simultaneously to the development of the present paper, M. Morini is approaching the problem in terms of counterparty risk [Mor08, [Mor09], F. Mercurio in terms of an extended Libor Market Model [Mer09], and M. Henrard using an axiomatic model [Hen09].

The present work follows an alternative route with respect to those cited above, in the sense that a) we adopt a bottom-up practitioner's perspective, starting from the current market practice of using multiple yield curves and working out its natural consequences,

\footnotetext{
${ }^{4}$ Forward Rate Agreement.

${ }^{5}$ these authors were puzzled by the fact that their first methodology was neither arbitrage free nor consistent with the pre-crisis single-curve market practice for pricing single-currency swaps. Such objections have now been overcome by the market evolution towards a generalized double-curve pricing approach (see also [TP03).
} 
looking for a minimal and light generalisation of well-known frameworks, keeping things as simple as possible; b) we show how no-arbitrage can be recovered in the double-curve approach by taking properly into account the forward basis, whose term structure can be extracted from available basis swap market quotations; c) we use a straightforward foreign-currency analogy to derive generalised double-curve market-like pricing expressions for basic single-currency interest rate derivatives, such as FRAs, swaps, caps/floors and swaptions.

\section{Notation and Basic Assumptions}

Following the discussion above, we denote with $M_{x}, x=\left\{d, f_{1}, \ldots, f_{n}\right\}$ multiple distinct interest rate sub-markets, characterized by the same currency and by distinct bank accounts $B_{x}$, such that

$$
B_{x}(t)=\exp \int_{0}^{t} r_{x}(u) d u
$$

where $r_{x}(t)$ are the associated short rates. We also have multiple yield curves $\complement_{x}$ in the form of a continuous term structure of discount factors,

$$
\complement_{x}=\left\{T \longrightarrow P_{x}\left(t_{0}, T\right), T \geq t_{0}\right\},
$$

where $t_{0}$ is the reference date of the curves (e.g. settlement date, or today) and $P_{x}(t, T)$ denotes the price at time $t \geq t_{0}$ of the $M_{x}$-zero coupon bond for maturity $T$, such that $P_{x}(T, T)=1$. In each sub-market $M_{x}$ we postulate the usual no arbitrage relation,

$$
P_{x}\left(t, T_{2}\right)=P_{x}\left(t, T_{1}\right) P_{x}\left(t, T_{1}, T_{2}\right), t \leq T_{1}<T_{2},
$$

where $P_{x}\left(t, T_{1}, T_{2}\right)$ denotes the $M_{x}$ forward discount factor from time $T_{2}$ to time $T_{1}$, prevailing at time $t$. The financial meaning of expression 3 is that, in each market $M_{x}$, given a cash flow of one unit of currency at time $T_{2}$, its corresponding value at time $t<T_{2}$ must be unique, both if we discount in one single step from $T_{2}$ to $t$, using the discount factor $P_{x}\left(t, T_{2}\right)$, and if we discount in two steps, first from $T_{2}$ to $T_{1}$, using the forward discount $P_{x}\left(t, T_{1}, T_{2}\right)$ and then from $T_{1}$ to $t$, using $P_{x}\left(t, T_{1}\right)$. Denoting with $F_{x}\left(t ; T_{1}, T_{2}\right)$ the simple compounded forward rate associated to $P_{x}\left(t, T_{1}, T_{2}\right)$, resetting at time $T_{1}$ and covering the time interval $\left[T_{1} ; T_{2}\right]$, we have

$$
P_{x}\left(t, T_{1}, T_{2}\right)=\frac{P_{x}\left(t, T_{2}\right)}{P_{x}\left(t, T_{1}\right)}=\frac{1}{1+F_{x}\left(t ; T_{1}, T_{2}\right) \tau_{x}\left(T_{1}, T_{2}\right)}
$$

where $\tau_{x}\left(T_{1}, T_{2}\right)$ is the year fraction between times $T_{1}$ and $T_{2}$ with daycount $d c_{x}$, and from eq. 3 we obtain the familiar no arbitrage expression

$$
\begin{aligned}
F_{x}\left(t ; T_{1}, T_{2}\right) & =\frac{1}{\tau_{x}\left(T_{1}, T_{2}\right)}\left[\frac{1}{P_{x}\left(t, T_{1}, T_{2}\right)}-1\right] \\
& =\frac{P_{x}\left(t, T_{1}\right)-P_{x}\left(t, T_{2}\right)}{\tau_{x}\left(T_{1}, T_{2}\right) P_{x}\left(t, T_{2}\right)} .
\end{aligned}
$$


Eq. 5 can be also derived (see e.g. ref. [BM06], sec. 1.4) as the fair value condition at time $t$ of the Forward Rate Agreement (FRA) contract with payoff at maturity $T_{2}$ given by

$$
\begin{gathered}
\operatorname{FRA}_{x}\left(T_{2} ; T_{1}, T_{2}, K, N\right)=N \tau_{x}\left(T_{1}, T_{2}\right)\left[L_{x}\left(T_{1}, T_{2}\right)-K\right] \\
L_{x}\left(T_{1}, T_{2}\right)=\frac{1-P_{x}\left(T_{1}, T_{2}\right)}{\tau_{x}\left(T_{1}, T_{2}\right) P_{x}\left(T_{1}, T_{2}\right)}
\end{gathered}
$$

where $N$ is the nominal amount, $L_{x}\left(T_{1}, T_{2}, d c_{x}\right)$ is the $T_{1}$-spot Xibor rate for maturity $T_{2}$ and $K$ the (simply compounded) strike rate (sharing the same daycount convention for simplicity). Introducing expectations we have, $\forall t \leq T_{1}<T_{2}$,

$$
\begin{aligned}
\operatorname{FRA}_{x}\left(t ; T_{1}, T_{2}, K, N\right) & =P_{x}\left(t, T_{2}\right) \mathbb{E}_{t}^{Q_{x}^{T_{2}}}\left[\mathbf{F R A}\left(T_{2} ; T_{1}, T_{2}, K, N\right)\right] \\
& =N P_{x}\left(t, T_{2}\right) \tau_{x}\left(T_{1}, T_{2}\right)\left\{\mathbb{E}_{t}^{Q_{x}^{T_{2}}}\left[L_{x}\left(T_{1}, T_{2}\right)\right]-K\right\} \\
& =N P_{x}\left(t, T_{2}\right) \tau_{x}\left(T_{1}, T_{2}\right)\left[F_{x}\left(t ; T_{1}, T_{2}\right)-K\right],
\end{aligned}
$$

where $Q_{x}^{T_{2}}$ denotes the $M_{x}-T_{2}$-forward measure corresponding to the numeraire $P_{x}\left(t, T_{2}\right)$, $\mathbb{E}_{t}^{Q}\left[\right.$.] denotes the expectation at time $t$ w.r.t. measure $Q$ and filtration $\mathcal{F}_{t}$, encoding the market information available up to time $t$, and we have assumed the standard martingale property of forward rates

$$
F_{x}\left(t ; T_{1}, T_{2}\right)=\mathbb{E}_{t}^{Q_{x}^{T_{2}}}\left[F_{x}\left(T_{1} ; T_{1}, T_{2}\right)\right]=\mathbb{E}_{t}^{Q_{x}^{T_{2}}}\left[L_{x}\left(T_{1}, T_{2}\right)\right]
$$

to hold in each interest rate market $M_{x}$ (see e.g. ref. [BM06]). We stress that the assumptions above imply that each sub-market $M_{x}$ is internally consistent and has the same properties of the "classical" interest rate market before the crisis. This is surely a strong hypothesis, that could be relaxed in more sophisticated frameworks.

\section{Pre and Post Credit Crunch Market Practices}

We describe here the evolution of the market practice for pricing and hedging interest rate derivatives through the credit crunch crisis. We use consistently the notation described above, considering a general single-currency interest rate derivative with $m$ future coupons with payoffs $\pi=\left\{\pi_{1}, \ldots, \pi_{m}\right\}$, with $\pi_{i}=\pi_{i}\left(F_{x}\right)$, generating $m$ cash flows $\mathbf{c}=\left\{c_{1}, \ldots, c_{m}\right\}$ at future dates $\mathbf{T}=\left\{T_{1}, \ldots, T_{m}\right\}$, with $t<T_{1}<\ldots<T_{m}$.

\subsection{Single-Curve Framework}

The pre-crisis standard market practice was based on a single-curve procedure, well known to the financial world, that can be summarised as follows (see e.g. refs. [Ron00], [HW06], [And07] and [HW08]):

1. select a single finite set of the most convenient (i.e. liquid) interest rate vanilla instruments traded on the market with increasing maturities and build a single yield curve $\complement_{d}$ using the preferred bootstrapping procedure (pillars, priorities, interpolation, etc.); for instance, a common choice in the EUR market is a combination of short term EUR deposits, medium-term Futures/FRA on Euribor3M and medium/long term swaps on Euribor6M; 
2. for each interest rate coupon $i \in\{1, \ldots, m\}$ compute the relevant forward rates using the given yield curve $\complement_{d}$ as in eq. 5 ,

$$
F_{d}\left(t ; T_{i-1}, T_{i}\right)=\frac{P_{d}\left(t, T_{i-1}\right)-P_{d}\left(t, T_{i}\right)}{\tau_{d}\left(T_{i-1}, T_{i}\right) P_{d}\left(t, T_{i}\right)} t \leq T_{i-1}<T_{i}
$$

3. compute cash flows $c_{i}$ as expectations at time $t$ of the corresponding coupon payoffs $\pi_{i}\left(F_{d}\right)$ with respect to the $T_{i}$-forward measure $Q_{d}^{T_{i}}$, associated to the numeraire $P_{d}\left(t, T_{i}\right)$ from the same yield curve $\complement_{d}$,

$$
c_{i}=c\left(t, T_{i}, \pi_{i}\right)=\mathbb{E}_{t}^{Q_{d}^{T_{i}}}\left[\pi_{i}\left(F_{d}\right)\right]
$$

4. compute the relevant discount factors $P_{d}\left(t, T_{i}\right)$ from the same yield curve $\complement_{d}$;

5. compute the derivative's price at time $t$ as the sum of the discounted cash flows,

$$
\pi(t ; \mathbf{T})=\sum_{i=1}^{m} P_{d}\left(t, T_{i}\right) c\left(t, T_{i}, \pi_{i}\right)=\sum_{i=1}^{m} P_{d}\left(t, T_{i}\right) \mathbb{E}_{t}^{Q_{d}^{T_{i}}}\left[\pi_{i}\left(F_{d}\right)\right]
$$

6. compute the delta sensitivity with respect to the market pillars of yield curve $\complement_{d}$ and hedge the resulting delta risk using the suggested amounts (hedge ratios) of the same set of vanillas.

For instance, a 5.5Y maturity EUR floating swap leg on Euribor1M (not directly quoted on the market) is commonly priced using discount factors and forward rates calculated on the same depo-Futures-swap curve cited above. The corresponding delta risk is hedged using the suggested amounts (hedge ratios) of $5 \mathrm{Y}$ and $6 \mathrm{Y}$ Euribor6M swap 6 .

Notice that step 3 above has been formulated in terms of the pricing measure $Q_{d}^{T_{i}}$ associated to the numeraire $P_{d}\left(t, T_{i}\right)$. This is convenient in our context because it emphasizes that the numeraire is associated to the discounting curve. Obviously any other equivalent measure associated to different numeraires may be used as well.

We stress that this is a single-currency-single-curve approach, in that a unique yield curve is built and used to price and hedge any interest rate derivative on a given currency. Thinking in terms of more fundamental variables, e.g. the short rate, this is equivalent to assume that there exist a unique fundamental underlying short rate process able to model and explain the whole term structure of interest rates of all tenors. It is also a relative pricing approach, because both the price and the hedge of a derivative are calculated relatively to a set of vanillas quoted on the market. We notice also that it is not strictly guaranteed to be arbitrage-free, because discount factors and forward rates obtained from a given yield curve through interpolation are, in general, not necessarily consistent with those obtained by a no arbitrage model; in practice bid-ask spreads and transaction costs hide any arbitrage possibilities. Finally, we stress that the key first point in the procedure is much more a matter of art than of science, because there is not an unique financially sound recipe for selecting the bootstrapping instruments and rules.

\footnotetext{
${ }^{6}$ we refer here to the case of local yield curve bootstrapping methods, for which there is no sensitivity delocalization effect (see refs. HW06], [HW08]).
} 


\subsection{Multiple-Curve Framework}

Unfortunately, the pre-crisis approach outlined above is no longer consistent, at least in its simple formulation, with the present market conditions. First, it does not take into account the market information carried by basis swap spreads, now much larger than in the past and no longer negligible. Second, it does not take into account that the interest rate market is segmented into sub-areas corresponding to instruments with distinct underlying rate tenors, characterized, in principle, by different dynamics (e.g. short rate processes). Thus, pricing and hedging an interest rate derivative on a single yield curve mixing different underlying rate tenors can lead to "dirty" results, incorporating the different dynamics, and eventually the inconsistencies, of distinct market areas, making prices and hedge ratios less stable and more difficult to interpret. On the other side, the more the vanillas and the derivative share the same homogeneous underlying rate, the better should be the relative pricing and the hedging. Third, by no arbitrage, discounting must be unique: two identical future cash flows of whatever origin must display the same present value; hence we need a unique discounting curve.

In principle, a consistent credit and liquidity theory would be required to account for the interest rate market segmentation. This would also explain the reason why the asymmetries cited above do not necessarily lead to arbitrage opportunities, once counterparty and liquidity risks are taken into account. Unfortunately such a framework is not easy to construct (see e.g. the discussion in refs. [Mer09, [Mor09]). In practice an empirical approach has prevailed on the market, based on the construction of multiple "forwarding" yield curves from plain vanilla market instruments homogeneous in the underlying rate tenor, used to calculate future cash flows based on forward interest rates with the corresponding tenor, and of a single "discounting" yield curve, used to calculate discount factors and cash flows' present values. Consequently, interest rate derivatives with a given underlying rate tenor should be priced and hedged using vanilla interest rate market instruments with the same underlying rate tenor. The post-crisis market practice may thus be summarised in the following working procedure:

1. build one discounting curve $\mathcal{\complement}_{d}$ using the preferred selection of vanilla interest rate market instruments and bootstrapping procedure;

2. build multiple distinct forwarding curves $\complement_{f_{1}}, \ldots, \complement_{f_{n}}$ using the preferred selections of distinct sets of vanilla interest rate market instruments, each homogeneous in the underlying Xibor rate tenor (typically with 1M, 3M, 6M, 12M tenors) and bootstrapping procedures;

3. for each interest rate coupon $i \in\{1, \ldots, m\}$ compute the relevant forward rates with tenor $f$ using the corresponding yield curve $\complement_{f}$ as in eq. 5,

$$
F_{f}\left(t ; T_{i-1}, T_{i}\right)=\frac{P_{f}\left(t, T_{i-1}\right)-P_{f}\left(t, T_{i}\right)}{\tau_{f}\left(T_{i-1}, T_{i}\right) P_{f}\left(t, T_{i}\right)}, \quad t \leq T_{i-1}<T_{i} ;
$$

4. compute cash flows $c_{i}$ as expectations at time $t$ of the corresponding coupon payoffs $\pi_{i}\left(F_{f}\right)$ with respect to the discounting $T_{i}$-forward measure $Q_{d}^{T_{i}}$, associated to the numeraire $P_{d}\left(t, T_{i}\right)$, as

$$
c_{i}=c\left(t, T_{i}, \pi_{i}\right)=\mathbb{E}_{t}^{Q_{d}^{T_{i}}}\left[\pi_{i}\left(F_{f}\right)\right]
$$


5. compute the relevant discount factors $P_{d}\left(t, T_{i}\right)$ from the discounting yield curve $\complement_{d}$;

6. compute the derivative's price at time $t$ as the sum of the discounted cash flows,

$$
\pi(t ; \mathbf{T})=\sum_{i=1}^{m} P_{d}\left(t, T_{i}\right) c\left(t, T_{i}, \pi_{i}\right)=\sum_{i=1}^{m} P_{d}\left(t, T_{i}\right) \mathbb{E}_{t}^{Q_{d}^{T_{i}}}\left[\pi_{i}\left(F_{f}\right)\right]
$$

7. compute the delta sensitivity with respect to the market pillars of each yield curve $\complement_{d}, \complement_{f_{1}}, \ldots, \complement_{f_{n}}$ and hedge the resulting delta risk using the suggested amounts (hedge ratios) of the corresponding set of vanillas.

For instance, the $5.5 \mathrm{Y}$ floating swap leg cited in the previous section 3.1 is currently priced using Euribor1M forward rates calculated on the $\complement_{1 M}$ forwarding curve, bootstrapped using Euribor1M vanillas only, plus discount factors calculated on the discounting curve $\complement_{d}$. The delta sensitivity is computed by shocking one by one the market pillars of both $\complement_{1 M}$ and $\complement_{d}$ curves and the resulting delta risk is hedged using the suggested amounts (hedge ratios) of $5 \mathrm{Y}$ and $6 \mathrm{Y}$ Euribor1M swaps plus the suggested amounts of $5 \mathrm{Y}$ and $6 \mathrm{Y}$ instruments from the discounting curve $\complement_{d}$ (see sec. 6.2 for more details about the hedging procedure).

Such multiple-curve framework is consistent with the present market situation, but there is no free lunch - it is also more demanding. First, the discounting curve clearly plays a special and fundamental role, and must be built with particular care. This "pre-crisis" obvious step has become, in the present market situation, a very subtle and controversial point, that would require a whole paper in itself (see e.g. ref. [Hen07]). In fact, while the forwarding curves construction is driven by the underlying rate homogeneity principle, for which there is (now) a general market consensus, there is no longer, at the moment, general consensus for the discounting curve construction. At least two different practices can be encountered in the market: a) the old "pre-crisis" approach (e.g. the depo, Futures/FRA and swap curve cited before), that can be justified with the principle of maximum liquidity (plus a little of inertia), and b) the OIS curve, based on the overnight rate (Eonia for EUR), considered as the best proxy to a risk free rate available on the market because of its 1-day tenor, justified with collateralized (riskless) counterparties 7 (see e.g. refs. [Mad08], GS009]). Second, building multiple curves requires multiple quotations: many more bootstrapping instruments must be considered (deposits, Futures, swaps, basis swaps, FRAs, etc., on different underlying rate tenors), which are available on the market with different degrees of liquidity and can display transitory inconsistencies (see [AB09]). Third, non trivial interpolation algorithms are crucial to produce smooth forward curves (see e.g. refs. [HW06]-[HW08], [AB09]). Fourth, multiple bootstrapping instruments implies multiple sensitivities, so hedging becomes more complicated. Last but not least, pricing libraries, platforms, reports, etc. must be extended, configured, tested and released to manage multiple and separated yield curves for forwarding and discounting, not a trivial task for quants, risk managers, developers and IT people.

\footnotetext{
${ }^{7}$ collateral agreements are more and more used in OTC markets, where there are no clearing houses, to reduce the counterparty risk. The standard ISDA contracts (ISDA Master Agreement and Credit Support Annex) include netting clauses imposing compensation. The compensation frequency is often on a daily basis and the (cash or asset) compensation amount is remunerated at overnight rate.
} 
The static multiple-curve pricing \& hedging methodology described above can be extended, in principle, by adopting multiple distinct models for the evolution of the underlying interest rates with tenors $f_{1}, \ldots, f_{n}$ to calculate the future dynamics of the yield curves $\complement_{f_{1}}, \ldots, \complement_{f_{n}}$ and the expected cash flows. The volatility/correlation dependencies carried by such models imply, in principle, bootstrapping multiple distinct variance/covariance matrices and hedging the corresponding sensitivities using volatility- and correlationdependent vanilla market instruments. Such more general approach has been carried on in ref. [Mer09] in the context of generalised market models. In this paper we will focus only on the basic matter of static yield curves and leave out the dynamical volatility/correlation dimensions.

\section{No Arbitrage and Forward Basis}

Now, we wish to understand the consequences of the assumptions above in terms of no arbitrage. First, we notice that, in the multiple-curve framework, classical single-curve no arbitrage relations are broken. For instance, if we assign index $d$ to discount factors and index $f$ to forward discount factors (containing forward rates) in eqs. 3 and 5 , we obtain

$$
\begin{gathered}
P_{f}\left(t, T_{1}, T_{2}\right)=\frac{P_{d}\left(t, T_{2}\right)}{P_{d}\left(t, T_{1}\right)}, \\
P_{f}\left(t, T_{1}, T_{2}\right)=\frac{1}{1+F_{f}\left(t ; T_{1}, T_{2}\right) \tau_{f}\left(T_{1}, T_{2}\right)}=\frac{P_{f}\left(t, T_{2}\right)}{P_{f}\left(t, T_{1}\right)},
\end{gathered}
$$

which are clearly inconsistent. No arbitrage between distinct yield curves $\complement_{d}$ and $\complement_{f}$ can be immediately recovered by taking into account the forward basis, the forward counterparty of the quoted market basis of fig. 1, defined as

$$
P_{f}\left(t, T_{1}, T_{2}\right):=\frac{1}{1+F_{d}\left(t ; T_{1}, T_{2}\right) B A_{f d}\left(t, T_{1}, T_{2}\right) \tau_{d}\left(T_{1}, T_{2}\right)}
$$

or through the equivalent simple transformation rule for forward rates

$$
F_{f}\left(t ; T_{1}, T_{2}\right) \tau_{f}\left(T_{1}, T_{2}\right)=F_{d}\left(t ; T_{1}, T_{2}\right) \tau_{d}\left(T_{1}, T_{2}\right) B A_{f d}\left(t, T_{1}, T_{2}\right) .
$$

From eq. 19 we can express the forward basis as a ratio between forward rates or, equivalently, in terms of discount factors from $\complement_{d}$ and $\complement_{f}$ curves as

$$
\begin{aligned}
B A_{f d}\left(t, T_{1}, T_{2}\right) & =\frac{F_{f}\left(t ; T_{1}, T_{2}\right) \tau_{f}\left(T_{1}, T_{2}\right)}{F_{d}\left(t ; T_{1}, T_{2}\right) \tau_{d}\left(T_{1}, T_{2}\right)} \\
& =\frac{P_{d}\left(t, T_{2}\right)}{P_{f}\left(t, T_{2}\right)} \frac{P_{f}\left(t, T_{1}\right)-P_{f}\left(t, T_{2}\right)}{P_{d}\left(t, T_{1}\right)-P_{d}\left(t, T_{2}\right)}
\end{aligned}
$$

Obviously the following alternative additive definition is completely equivalent

$$
P_{f}\left(t, T_{1}, T_{2}\right):=\frac{1}{1+\left[F_{d}\left(t ; T_{1}, T_{2}\right)+B A_{f d}^{\prime}\left(t, T_{1}, T_{2}\right)\right] \tau_{d}\left(T_{1}, T_{2}\right)},
$$




$$
\begin{aligned}
B A_{f d}^{\prime}\left(t, T_{1}, T_{2}\right) & =\frac{F_{f}\left(t ; T_{1}, T_{2}\right) \tau_{f}\left(T_{1}, T_{2}\right)-F_{d}\left(t ; T_{1}, T_{2}\right) \tau_{d}\left(T_{1}, T_{2}\right)}{\tau_{d}\left(T_{1}, T_{2}\right)} \\
& =\frac{1}{\tau_{d}\left(T_{1}, T_{2}\right)}\left[\frac{P_{f}\left(t, T_{1}\right)}{P_{f}\left(t, T_{2}\right)}-\frac{P_{d}\left(t, T_{1}\right)}{P_{d}\left(t, T_{2}\right)}\right] \\
& =F_{d}\left(t ; T_{1}, T_{2}\right)\left[B A_{f d}\left(t, T_{1}, T_{2}\right)-1\right],
\end{aligned}
$$

which is more useful for comparisons with the market basis spreads of fig. 1. Notice that if $\complement_{d}=\complement_{f}$ we recover the single-curve case $B A_{f d}\left(t, T_{1}, T_{2}\right)=1, B A_{f d}^{\prime}\left(t, T_{1}, T_{2}\right)=0$.

We stress that the forward basis in eqs. 20,22 is a straightforward consequence of the assumptions above, essentially the existence of two yield curves and no arbitrage. Its advantage is that it allows for a direct computation of the forward basis between forward rates for any time interval $\left[T_{1}, T_{2}\right]$, which is the relevant quantity for pricing and hedging interest rate derivatives. In practice its value depends on the market basis spread between the quotations of the two sets of vanilla instruments used in the bootstrapping of the two curves $\complement_{d}$ and $\complement_{f}$. On the other side, the limit of expressions $20+22$ is that they reflect the statica 8 differences between the two interest rate markets $M_{d}, M_{f}$ carried by the two curves $\complement_{d}, \complement_{f}$, but they are completely independent of the interest rate dynamics in $M_{d}$ and $M_{f}$.

Notice also that the approach can be inverted to bootstrap a new yield curve from a given yield curve plus a given forward basis, using the following recursive relations

$$
\begin{aligned}
P_{d, i} & =\frac{P_{f, i} B A_{f d, i}}{P_{f, i-1}-P_{f, i}+P_{f, i} B A_{f d, i}} P_{d, i-1} \\
& =\frac{P_{f, i}}{P_{f, i-1}-P_{f, i} B A_{f d, i}^{\prime} \tau_{d, i}} P_{d, i-1}, \\
P_{f, i} & =\frac{P_{d, i}}{P_{d, i}+\left(P_{d, i-1}-P_{d, i}\right) B A_{f d, i}} P_{f, i-1} \\
& =\frac{P_{d, i}}{P_{d, i}+P_{d, i-1} B A_{f d, i}^{\prime} \tau_{d, i}} P_{f, i-1},
\end{aligned}
$$

where we have inverted eqs. 20, 22 and shortened the notation by putting $\tau_{x}\left(T_{i-1}, T_{i}\right):=$ $\tau_{x, i}, P_{x}\left(t, T_{i}\right):=P_{x, i}, B A_{f d}\left(t, T_{i-1}, T_{i}\right):=B A_{f d, i}$. Given the yield curve $x$ up to step $P_{x, i-1}$ plus the forward basis for the step $i-1 \rightarrow i$, the equations above can be used to obtain the next step $P_{x, i}$.

We now discuss a numerical example of the forward basis in a realistic market situation. We consider the four interest rate underlyings $\mathbf{I}=\left\{I_{1 M}, I_{3 M}, I_{6 M}, I_{12 M}\right\}$, where $I=$ Euribor index, and we bootstrap from market data five distinct yield curves $\complement=\left\{\complement_{d}\right.$, $\left.\complement_{1 M}, \complement_{3 M}, \complement_{6 M}, \complement_{12 M}\right\}$, using the first one for discounting and the others for forwarding. We follow the methodology described in ref. AB09 using the corresponding open-source development available in the QuantLib framework Qua09. The discounting curve $\complement_{d}$ is built following a "pre-crisis" traditional recipe from the most liquid deposit, IMM Futures/FRA on Euribor3M and swaps on Euribor6M. The other four forwarding curves are built from convenient selections of depos, FRAs, Futures, swaps and basis swaps with homogeneous underlying rate tenors; a smooth and robust algorithm (monotonic

\footnotetext{
${ }^{8}$ we remind that the discount factors in eqs. 20,20 are calculated on the curves $\complement_{d}, \complement_{f}$ following the recipe described in sec. 3.2, not using any dynamical model for the evolution of the rates.
} 

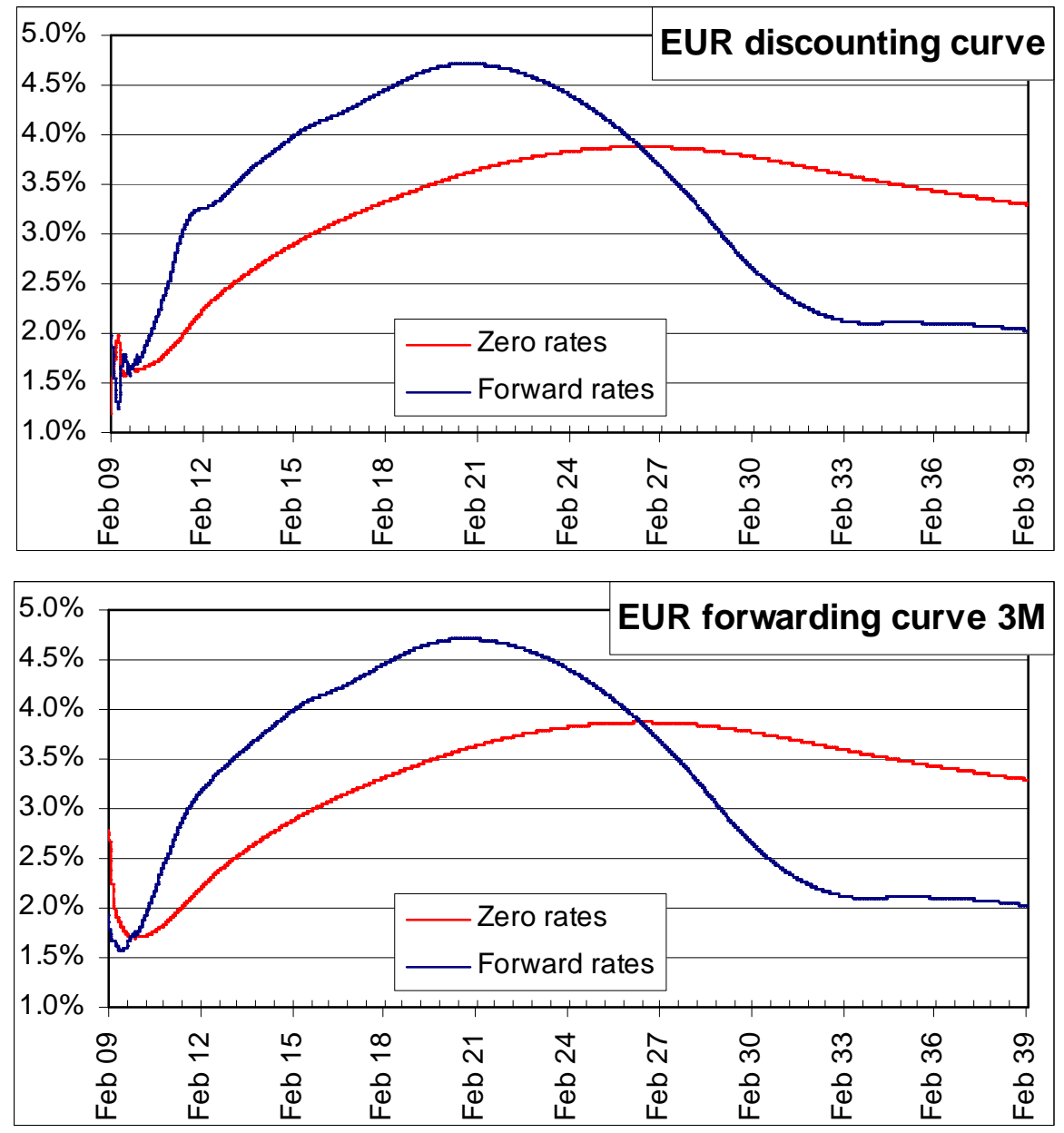

Figure 2: EUR discounting curve $\complement_{d}$ (upper panel) and $3 \mathrm{M}$ forwarding curve $\complement_{3 M}$ (lower panel) at end of day Feb. 16th 2009. Blue lines: 3M-tenor forward rates $F\left(t_{0} ; t, t+3 M\right.$, act/360 $), t$ daily sampled and spot date $t_{0}=18$ th Feb. 2009; red lines: zero rates $F\left(t_{0} ; t\right.$, act/365). Similar patterns are observed also in the $1 \mathrm{M}, 6 \mathrm{M}, 12 \mathrm{M}$ curves (not shown here, see ref. [AB09]).

cubic spline on log discounts) is used for interpolations. Different choices (e.g. an Eonia discounting curve) as well as other technicalities of the bootstrapping described in ref. [AB09] obviously would lead to slightly different numerical results, but do not alter the conclusions drawn here.

In fig. 2 we plot both the 3M-tenor forward rates and the zero rates calculated on $\complement_{d}$ and $\complement_{3 M}$ as of 16th Feb. 2009 cob 9 . Similar patterns are observed also in the other $1 \mathrm{M}, 6 \mathrm{M}, 12 \mathrm{M}$ curves (not shown here, see ref. AB09). In fig. 3 (upper panels) we plot the term structure of the four corresponding multiplicative forward basis curves $\complement_{f}-\complement_{d}$ calculated through eq. 20, In the lower panels we also plot the additive forward basis given by eq. 22. We observe in particular that the higher short-term basis adjustments (left panels) are due to the higher short-term market basis spreads (see fig. 11). Furthermore, the medium-long-term $\complement_{6 M}-\complement_{d}$ basis (dash-dotted green lines in the right panels) are

\footnotetext{
${ }^{9}$ close of business.
} 

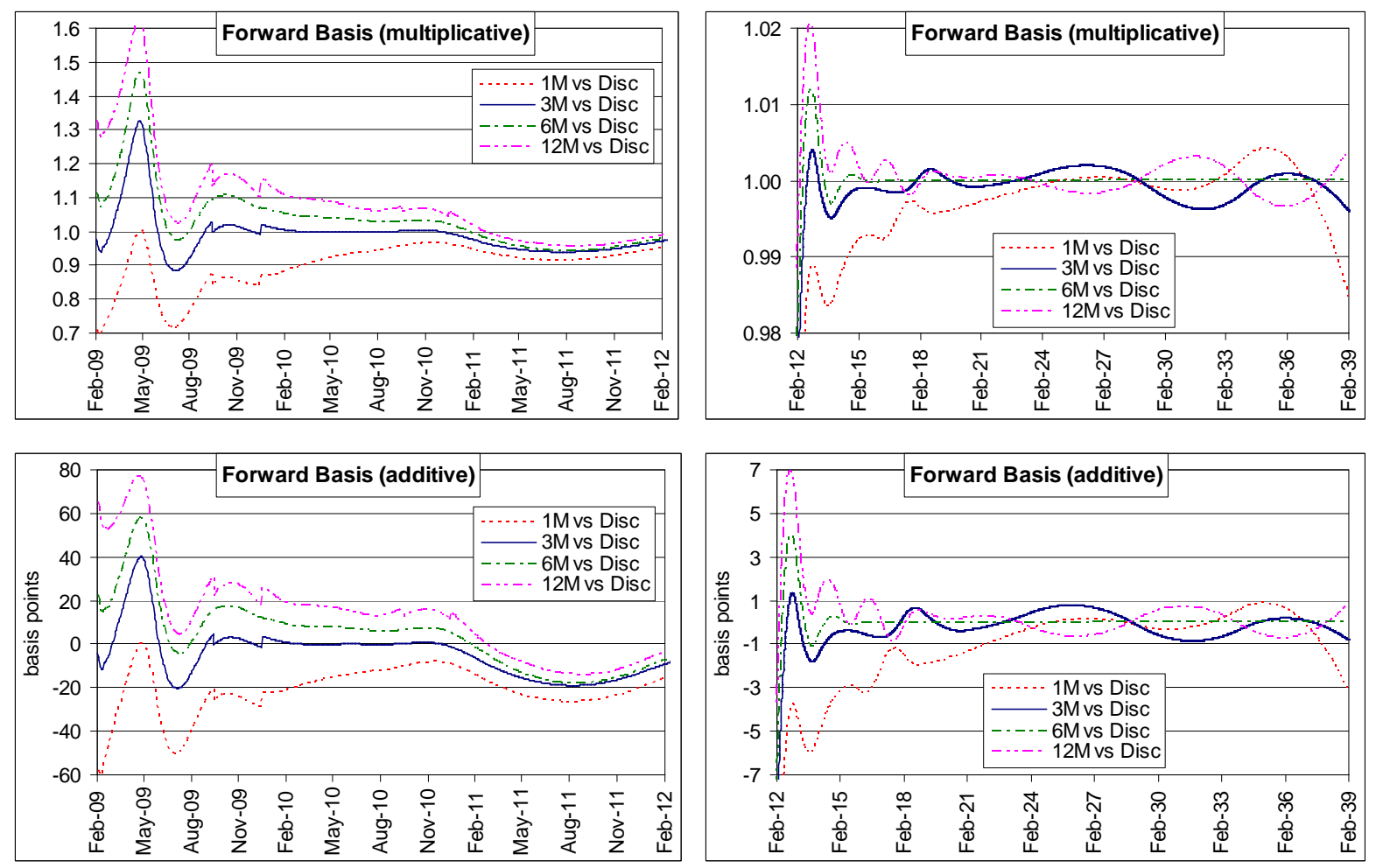

Figure 3: upper panels: multiplicative basis adjustments from eq. 20 as of end of day Feb. 16th, 2009, for daily sampled 3M-tenor forward rates as in fig. 2, calculated on $\complement_{1 M}$, $\complement_{3 M}, \complement_{6 M}$ and $\complement_{12 M}$ curves against $\complement_{d}$ taken as reference curve. Lower panels: equivalent plots of the additive basis adjustment of eq. 22 between the same forward rates (basis points). Left panels: 0Y-3Y data; Right panels: 3Y-30Y data on magnified scales. The higher short-term adjustments seen in the left panels are due to the higher short-term market basis spread (see Figs. 1). The oscillating term structure observed is due to the amplification of small differences in the term structures of the curves.

close to 1 and 0 , respectively, as expected from the common use of $6 \mathrm{M}$ swaps in the two curves. A similar, but less evident, behavior is found in the short-term $\complement_{3 M}-\complement_{d}$ basis (continuous blue line in the left panels), as expected from the common 3M Futures and the uncommon deposits. The two remaining basis curves $\complement_{1 M}-\complement_{d}$ and $\complement_{12 M}-\complement_{d}$ are generally far from 1 or 0 because of different bootstrapping instruments. Obviously such details depend on our arbitrary choice of the discounting curve.

Overall, we notice that all the basis curves $\complement_{f}-\complement_{d}$ reveal a complex micro-term structure, not present either in the monotonic basis swaps market quotes of fig. 1 or in the smooth yield curves $\complement_{x}$. Such effect is essentially due to an amplification mechanism of small local differences between the $\complement_{d}$ and $\complement_{f}$ forward curves. In fig. 4 we also show that smooth yield curves are a crucial input for the forward basis: using a non-smooth bootstrapping (linear interpolation on zero rates, still a diffused market practice), the zero curve apparently shows no particular problems, while the forward curve displays a sagsaw shape inducing, in turn, strong and unnatural oscillations in the forward basis.

We conclude that, once a smooth and robust bootstrapping technique for yield curve construction is used, the richer term structure of the forward basis curves provides a 

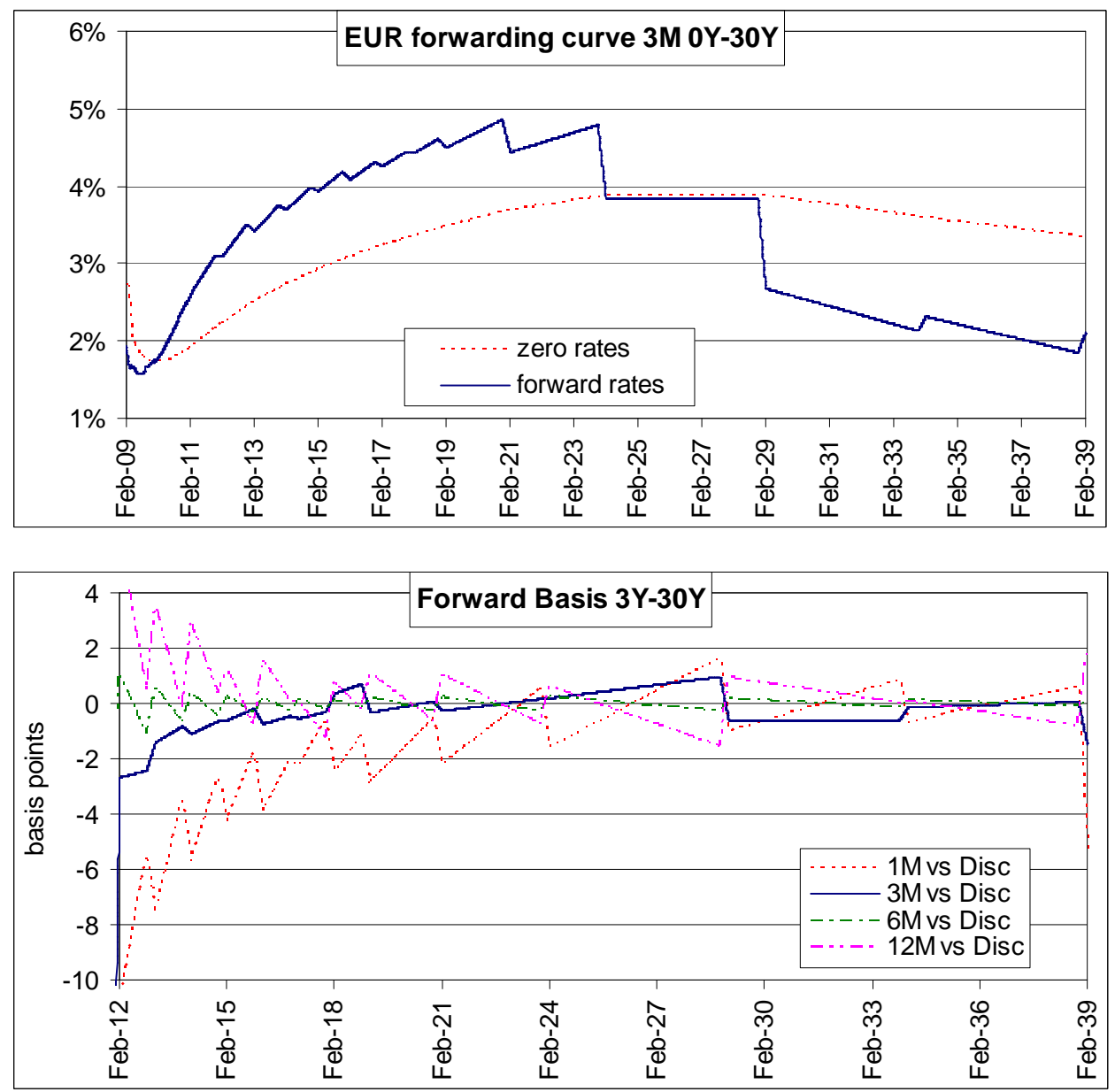

Figure 4: the effect of poor interpolation schemes (linear on zero rates, a common choice, see ref. [AB09]) on zero rates (upper panel, red line) 3M forward rates (upper panel, blue line) and basis adjustments (lower panel). While the zero curve looks smooth, the sag-saw shape of the forward curve clearly show the inadequacy of the bootstrap, and the oscillations in the basis adjustment allow to further appreciate the artificial differences induced in similar instruments priced on the two curves.

sensitive indicator of the tiny, but observable, statical differences between different interest rate market sub-areas in the post credit crunch interest rate world, and a tool to assess the degree of liquidity and credit issues in interest rate derivatives' prices. It is also helpful for a better explanation of the profit\&loss encountered when switching between the singleand the multiple-curve worlds.

\section{Foreign-Currency Analogy and Quanto Adjustment}

A second important issue regarding no-arbitrage arises in the multiple-curve framework. From eq. 14 we have that, for instance, the single-curve FRA price in eq. 8 is generalised 
into the following multiple-curve expression

$$
\operatorname{FRA}\left(t ; T_{1}, T_{2}, K, N\right)=N P_{d}\left(t, T_{2}\right) \tau_{f}\left(T_{1}, T_{2}\right)\left\{\mathbb{E}_{t}^{Q_{d}^{T_{2}}}\left[L_{f}\left(T_{1}, T_{2}\right)\right]-K\right\}
$$

Instead, the current market practice is to price such FRA simply as

$$
\operatorname{FRA}\left(t ; T_{1}, T_{2}, K, N\right) \simeq N P_{d}\left(t, T_{2}\right) \tau_{f}\left(T_{1}, T_{2}\right)\left[F_{f}\left(t ; T_{1}, T_{2}\right)-K\right]
$$

Obviously the forward rate $F_{f}\left(t ; T_{1}, T_{2}\right)$ is not, in general, a martingale under the discounting measure $Q_{d}^{T_{2}}$, thus eq. 26] is just an approximation that discards the adjustment coming from this measure mismatch. Hence, a theoretically correct pricing within the multiple-curve framework requires the computation of expectations as in eq. 25 above. This will involve the dynamic properties of the two interest rate markets $M_{d}$ and $M_{f}$, or, in other words, it will require to model the dynamics for the interest rates in $M_{d}$ and $M_{f}$. This task is easily accomplished by resorting to the natural analogy with cross-currency derivatives. Going back to the beginning of sec. 2, we can identify $M_{d}$ and $M_{f}$ with the domestic and foreign markets, $\complement_{d}$ and $\complement_{f}$ with the corresponding curves, and the bank accounts $B_{d}(t), B_{f}(t)$ with the corresponding currencies, respectively 10 . Within this framework, we can recognize on the r.h.s of eq. 18 the forward discount factor from time $T_{2}$ to time $T_{1}$ expressed in domestic currency, and on the r.h.s. of eq. 25 the expectation of the foreign forward rate w.r.t the domestic forward measure. Hence, the computation of such expectation must involve the quanto adjustment commonly encountered in the pricing of cross-currency derivatives. The derivation of such adjustment can be found in standard textbooks. Anyway, in order to fully appreciate the parallel with the present double-curve-single-currency case, it is useful to run through it once again. In particular, we will adapt to the present context the discussion found in ref. [BM06], chs. 2.9 and 14.4 .

\section{$5.1 \quad$ Forward Rates}

In the double-curve-double-currency case, no arbitrage requires the existence at any time $t_{0} \leq t \leq T$ of a spot and a forward exchange rate between equivalent amounts of money in the two currencies such that

$$
\begin{gathered}
c_{d}(t)=x_{f d}(t) c_{f}(t), \\
X_{f d}(t, T) P_{d}(t, T)=x_{f d}(t) P_{f}(t, T),
\end{gathered}
$$

where the subscripts $f$ and $d$ stand for foreign and domestic, $c_{d}(t)$ is any cash flow (amount of money) at time $t$ in units of domestic-currency and $c_{f}(t)$ is the corresponding cash flow at time $t$ (the corresponding amount of money) in units of foreign currency. Obviously $X_{f d}(t, T) \rightarrow x_{f d}(t)$ for $t \rightarrow T$. Expression 28 is still a consequence of no arbitrage. This can be understood with the aid of fig. 5; starting from top right corner in the time vs currency/yield curve plane with an unitary cash flow at time $T>t$ in foreign currency, we can either move along path A by discounting at time $t$ on curve $\complement_{f}$ using

\footnotetext{
${ }^{10}$ notice the lucky notation used, where " $d$ " stands either for "discounting" or "domestic" and " $f$ " for "forwarding" or "foreign", respectively.
} 


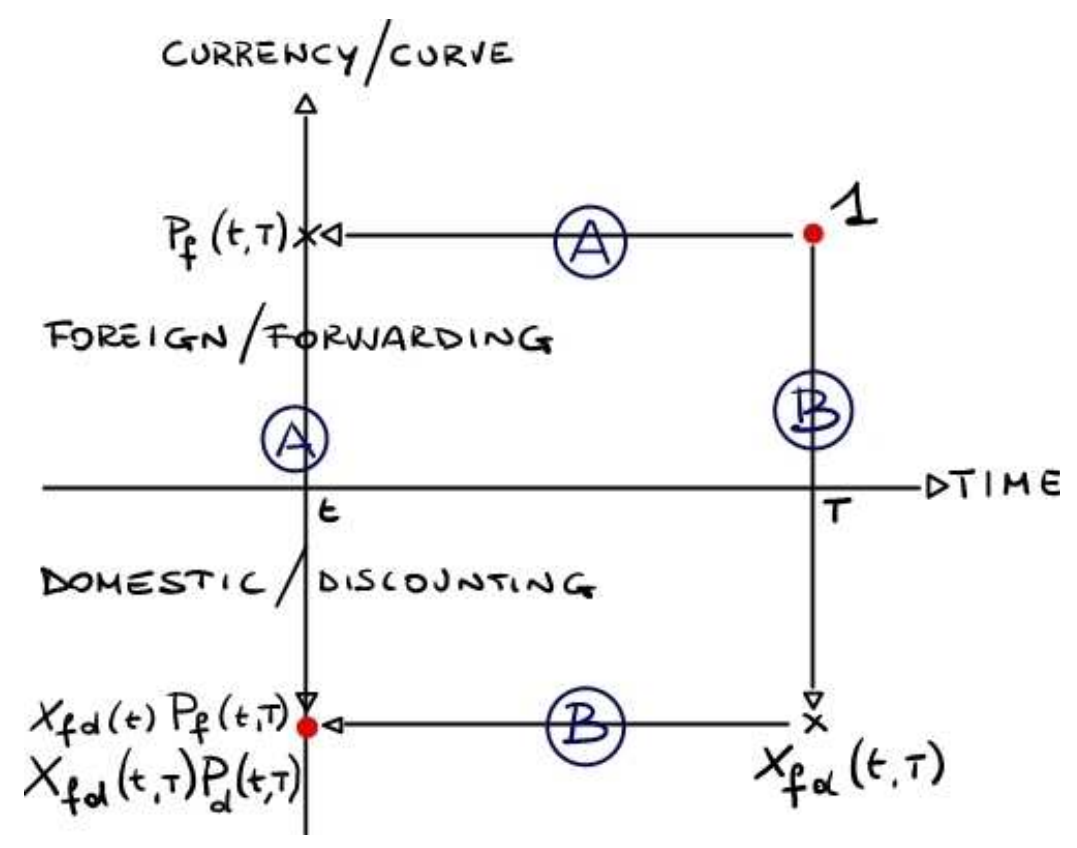

Figure 5: Picture of no-arbitrage interpretation for the forward exchange rate in eq. 28, Moving, in the yield curve vs time plane, from top right to bottom left corner through path $\mathrm{A}$ or path $\mathrm{B}$ must be equivalent. Alternatively, we may think to no-arbitrage as a sort of zero "circuitation", sum of all trading events following a closed path starting and stopping at the same point in the plane. This description is equivalent to the traditional "table of transaction" picture, as found e.g. in fig. 1 of ref. [TP03].

$P_{f}(t, T)$ and then by changing into domestic currency units using the spot exchange rate $x_{f d}(t)$, ending up with $x_{f d}(t) P_{f}(t, T)$ units of domestic currency; or, alternatively, we can follow path $\mathrm{B}$ by changing at time $T$ into domestic currency units using the forward exchange rate $X_{f d}(t, T)$ and then by discounting on $\complement_{d}$ using $P_{d}(t, T)$, ending up with $X_{f d}(t, T) P_{d}(t, T)$ units of domestic currency. Both paths stop at bottom left corner, hence eq. 28 must hold by no arbitrage.

Now, our double-curve-single-currency case is immediately obtained from the discussion above by thinking to the subscripts $f$ and $d$ as shorthands for forwarding and discounting and by recognizing that, having a single currency, the today's spot exchange rate must collapse to $1, x_{f d}\left(t_{0}\right)=1$. Obviously for $\complement_{d}=\complement_{f}$ we recover the single-currency, single-curve case $X_{f d}(t, T)=1 \forall t, T$. The financial interpretation of the forward exchange rate in eq. 28 within this framework is straightforward: it is nothing else that the counterparty of the forward basis in eq. 19 for discount factors on the two yield curves $\complement_{d}$ and $\complement_{f}$. Substituting eq. 28 into eq. 19 we obtain the following relation

$$
B A_{f d}\left(t, T_{1}, T_{2}\right)=\frac{P_{d}\left(t, T_{1}\right) X_{f d}\left(t, T_{1}\right)-P_{d}\left(t, T_{2}\right) X_{f d}\left(t, T_{2}\right)}{X_{f d}\left(t, T_{2}\right)\left[P_{d}\left(t, T_{1}\right)-P_{d}\left(t, T_{2}\right)\right]} .
$$

We proceed by assuming, according to the standard market practice, the following (driftless) lognormal martingale dynamic for $\complement_{f}$ (foreign) forward rates

$$
\frac{d F_{f}\left(t ; T_{1}, T_{2}\right)}{F_{f}\left(t ; T_{1}, T_{2}\right)}=\sigma_{f}(t) d W_{f}^{T_{2}}(t), \quad t \leq T_{1}
$$


where $\sigma_{f}(t)$ is the volatility (positive deterministic function of time) of the process, under the probability space $\left(\Omega, \mathcal{F}^{f}, Q_{f}^{T_{2}}\right)$ with the filtration $\mathcal{F}_{t}^{f}$ generated by the brownian motion $W_{f}^{T_{2}}$ under the forwarding (foreign) $T_{2}$-forward measure $Q_{f}^{T_{2}}$, associated to the $\complement_{f}$ (foreign) numeraire $P_{f}\left(t, T_{2}\right)$.

Next, since $X_{f d}\left(t, T_{2}\right)$ in eq. 28 is the ratio between the price at time $t$ of a $\complement_{d}$ (domestic) tradable asset $x_{f d}(t) P_{f}\left(t, T_{2}\right)$ and the $\complement_{d}$ numeraire $P_{d}\left(t, T_{2}\right)$, it must evolve according to a (driftless) martingale process under the associated discounting (domestic) $T_{2}$ - forward measure $Q_{d}^{T_{2}}$,

$$
\frac{d X_{f d}\left(t, T_{2}\right)}{X_{f d}\left(t, T_{2}\right)}=\sigma_{X}(t) d W_{X}^{T_{2}}(t), \quad t \leq T_{2}
$$

where $\sigma_{X}(t)$ is the volatility (positive deterministic function of time) of the process and $W_{X}^{T_{2}}$ is a brownian motion under $Q_{d}^{T_{2}}$ such that

$$
d W_{f}^{T_{2}}(t) d W_{X}^{T_{2}}(t)=\rho_{f X}(t) d t
$$

Now, in order to calculate expectations such as in the r.h.s. of eq. 25, we must switch from the forwarding (foreign) measure $Q_{f}^{T_{2}}$ associated to the numeraire $P_{f}\left(t, T_{2}\right)$ to the discounting (domestic) measure $Q_{d}^{T_{2}}$ associated to the numeraire $P_{d}\left(t, T_{2}\right)$. In our doublecurve-single-currency language this amounts to transform a cash flow on curve $\complement_{f}$ to the corresponding cash flow on curve $\complement_{d}$. Recurring to the change-of-numeraire technique (see refs. [BM06], [Jam89], [GKR95]) we obtain that the dynamic of $F_{f}\left(t ; T_{1}, T_{2}\right)$ under $Q_{d}^{T_{2}}$ acquires a non-zero drift

$$
\begin{gathered}
\frac{d F_{f}\left(t ; T_{1}, T_{2}\right)}{F_{f}\left(t ; T_{1}, T_{2}\right)}=\mu_{f}(t) d t+\sigma_{f}(t) d W_{f}^{T_{2}}(t), \quad t \leq T_{1}, \\
\mu_{f}(t)=-\sigma_{f}(t) \sigma_{X}(t) \rho_{f X}(t)
\end{gathered}
$$

and that $F_{f}\left(T_{1} ; T_{1}, T_{2}\right)$ is lognormally distributed under $Q_{d}^{T_{2}}$ with mean and variance given by

$$
\begin{gathered}
\mathbb{E}_{t}^{Q_{d}^{T_{2}}}\left[\ln \frac{F_{f}\left(T_{1} ; T_{1}, T_{2}\right)}{F_{f}\left(t ; T_{1}, T_{2}\right)}\right]=\int_{t}^{T_{1}}\left[\mu_{f}(u)-\frac{1}{2} \sigma_{f}^{2}(u)\right] d u \\
\operatorname{Var}_{t}^{Q_{d}^{T_{2}}}\left[\ln \frac{F_{f}\left(T_{1} ; T_{1}, T_{2}\right)}{F_{f}\left(t ; T_{1}, T_{2}\right)}\right]=\int_{t}^{T_{1}} \sigma_{f}^{2}(u) d u
\end{gathered}
$$

We thus obtain the following expressions, for $t_{0} \leq t<T_{1}$,

$$
\begin{aligned}
\mathbb{E}_{t}^{Q_{d}^{T_{2}}}\left[F_{f}\left(T_{1} ; T_{1}, T_{2}\right)\right] & =F_{f}\left(t ; T_{1}, T_{2}\right) Q A_{f d}\left(t, T_{1}, \sigma_{f}, \sigma_{X}, \rho_{f X}\right), \\
Q A_{f d}\left(t, T_{1}, \sigma_{f}, \sigma_{X}, \rho_{f X}\right) & =\exp \int_{t}^{T_{1}} \mu_{f}(u) d u \\
& =\exp \left[-\int_{t}^{T_{1}} \sigma_{f}(u) \sigma_{X}(u) \rho_{f X}(u) d u\right]
\end{aligned}
$$


where $Q A_{f d}\left(t, T_{1}, \sigma_{f}, \sigma_{X}, \rho_{f X}\right)$ is the (multiplicative) quanto adjustment. We may also define an additive quanto adjustment as

$$
\begin{aligned}
\mathbb{E}_{t}^{Q_{d}^{T_{2}}}\left[F_{f}\left(T_{1} ; T_{1}, T_{2}\right)\right] & =F_{f}\left(t ; T_{1}, T_{2}\right)+Q A_{f d}^{\prime}\left(t, T_{1}, \sigma_{f}, \sigma_{X}, \rho_{f X}\right), \\
Q A_{f d}^{\prime}\left(t, T_{1}, \sigma_{f}, \sigma_{X}, \rho_{f X}\right) & =F_{f}\left(t ; T_{1}, T_{2}\right)\left[Q A_{f d}\left(t, T_{1}, \sigma_{f}, \sigma_{X}, \rho_{f X}\right)-1\right],
\end{aligned}
$$

where the second relation comes from eq. 37. Finally, combining eqs. 37, 39 with eqs. 20, 22 we may derive a relation between the quanto and the basis adjustments,

$$
\begin{gathered}
B A_{f d}\left(t, T_{1}, T_{2}\right) Q A_{f d}\left(t, T_{1}, \sigma_{f}, \sigma_{X}, \rho_{f X}\right)=\frac{\tau_{f}\left(T_{1}, T_{2}\right) \mathbb{E}_{t}^{Q_{d}^{T_{2}}}\left[L_{f}\left(T_{1}, T_{2}\right)\right]}{\tau_{d}\left(T_{1}, T_{2}\right) \mathbb{E}_{t}^{Q_{d}^{T_{2}}}\left[L_{d}\left(T_{1}, T_{2}\right)\right]}, \\
B A_{f d}^{\prime}\left(t, T_{1}, T_{2}\right) \tau_{d}\left(T_{1}, T_{2}\right)+Q A_{f d}^{\prime}\left(t, T_{1}, \sigma_{f}, \sigma_{X}, \rho_{f X}\right) \tau_{f}\left(T_{1}, T_{2}\right) \\
=\mathbb{E}_{t}^{Q_{d}^{T_{2}}}\left[L_{f}\left(T_{1}, T_{2}\right)\right] \tau_{f}\left(T_{1}, T_{2}\right)-\mathbb{E}_{t}^{Q_{d}^{T_{2}}}\left[L_{d}\left(T_{1}, T_{2}\right)\right] \tau_{d}\left(T_{1}, T_{2}\right)
\end{gathered}
$$

for multiplicative and additive adjustments, respectively.

We conclude that the foreign-currency analogy allows us to compute the expectation in eq. 25 of a forward rate on curve $\complement_{f}$ w.r.t. the discounting measure $Q_{d}^{T_{2}}$ in terms of a well-known quanto adjustment, typical of cross-currency derivatives. Such adjustment naturally follows from a change between the $T$-forward probability measures $Q_{f}^{T_{2}}$ and $Q_{d}^{T_{2}}$, or numeraires $P_{f}\left(t, T_{2}\right)$ and $P_{d}\left(t, T_{2}\right)$, associated to the two yield curves, $\complement_{f}$ and $\complement_{d}$, respectively. Notice that the expression 38 depends on the average over the time interval $\left[t, T_{1}\right]$ of the product of the volatility $\sigma_{f}$ of the $\complement_{f}$ (foreign) forward rates $F_{f}$, of the volatility $\sigma_{X}$ of the forward exchange rate $X_{f d}$ between curves $\complement_{f}$ and $\complement_{d}$, and of the correlation $\rho_{f X}$ between $F_{f}$ and $X_{f d}$. It does not depend either on the volatility $\sigma_{d}$ of the $\complement_{d}$ (domestic) forward rates $F_{d}$ or on any stochastic quantity after time $T_{1}$. The latter fact is actually quite natural, because the stochasticity of the forward rates involved ceases at their fixing time $T_{1}$. The dependence on the cash flow time $T_{2}$ is actually implicit in eq 38, because the volatilities and the correlation involved are exactly those of the forward and exchange rates on the time interval $\left[T_{1}, T_{2}\right]$. Notice in particular that a non-trivial adjustment is obtained if and only if the forward exchange rate $X_{f d}$ is stochastic $\left(\sigma_{X} \neq 0\right)$ and correlated to the forward rate $F_{f}\left(\rho_{f X} \neq 0\right)$; otherwise expression 38 collapses to the single curve case $Q A_{f d}=1$.

\subsection{Swap Rates}

The discussion above can be remapped, with some attention, to swap rates. Given two increasing dates sets $\mathbf{T}=\left\{T_{0}, \ldots, T_{n}\right\}, \mathbf{S}=\left\{S_{0}, \ldots, S_{m}\right\}, T_{0}=S_{0} \geq t$ and an interest rate swap with a floating leg paying at times $T_{i}, i=1, . ., n$, the Xibor rate with tenor $\left[T_{i-1}, T_{i}\right]$ fixed at time $T_{i-1}$, plus a fixed leg paying at times $S_{j}, j=1, . ., m$, a fixed rate, the corresponding fair swap rate $S_{f}(t, \mathbf{T}, \mathbf{S})$ on curve $\complement_{f}$ is defined by the following equilibrium (no arbitrage) relation between the present values of the two legs,

$$
S_{f}(t, \mathbf{T}, \mathbf{S}) A_{f}(t, \mathbf{S})=\sum_{i=1}^{n} P_{f}\left(t, T_{i}\right) \tau_{f}\left(T_{i-1}, T_{i}\right) F_{f}\left(t ; T_{i-1}, T_{i}\right), \quad t \leq T_{0}=S_{0}
$$


where

$$
A_{f}(t, \mathbf{S})=\sum_{j=1}^{m} P_{f}\left(t, S_{j}\right) \tau_{f}\left(S_{j-1}, S_{j}\right)
$$

is the annuity on curve $\complement_{f}$. Following the standard market practice, we observe that, assuming the annuity as the numeraire on curve $\complement_{f}$, the swap rate in eq. 43 is the ratio between a tradable asset (the value of the swap floating leg on curve $\complement_{f}$ ) and the numeraire $A_{f}(t, \mathbf{S})$, and thus it is a martingale under the associated forwarding (foreign) swap measure $Q_{f}^{\mathbf{S}}$. Hence we can assume, as in eq. 30, a driftless geometric brownian motion for the swap rate under $Q_{f}^{\mathbf{S}}$,

$$
\frac{d S_{f}(t, \mathbf{T}, \mathbf{S})}{S_{f}(t, \mathbf{T}, \mathbf{S})}=\nu_{f}(t, \mathbf{T}, \mathbf{S}) d W_{f}^{\mathbf{T}, \mathbf{S}}(t), \quad t \leq T_{0},
$$

where $v_{f}(t, \mathbf{T}, \mathbf{S})$ is the volatility (positive deterministic function of time) of the process and $W_{f}^{\mathbf{T}, \mathbf{S}}$ is a brownian motion under $Q_{f}^{\mathbf{S}}$. Then, mimicking the discussion leading to eq. 28, the following relation

$$
\begin{aligned}
\sum_{j=1}^{m} P_{d}\left(t, S_{j}\right) \tau_{d}\left(S_{j-1}, S_{j}\right) X_{f d}\left(t, S_{j}\right) & =x_{f d}(t) \sum_{j=1}^{m} P_{f}\left(t, S_{j}\right) \tau_{f}\left(S_{j-1}, S_{j}\right) \\
& =x_{f d}(t) A_{f}(t, \mathbf{S})
\end{aligned}
$$

must hold by no arbitrage between the two curves $\complement_{f}$ and $\complement_{d}$. Defining a swap forward exchange rate $Y_{f d}(t, \mathbf{S})$ such that

$$
\begin{aligned}
x_{f d}(t) A_{f}(t, \mathbf{S}), & =\sum_{j=1}^{m} P_{d}\left(t, S_{j}\right) \tau_{d}\left(S_{j-1}, S_{j}\right) X_{f d}\left(t, S_{j}\right) \\
& =Y_{f d}(t, \mathbf{S}) \sum_{j=1}^{m} P_{d}\left(t, S_{j}\right) \tau_{d}\left(S_{j-1}, S_{j}\right)=Y_{f d}(t, \mathbf{S}) A_{d}(t, \mathbf{S}),
\end{aligned}
$$

we obtain the expression

$$
Y_{f d}(t, \mathbf{S})=x_{f d}(t) \frac{A_{f}(t, \mathbf{S})}{A_{d}(t, \mathbf{S})},
$$

equivalent to eq. 28. Hence, since $Y_{f d}(t, \mathbf{S})$ is the ratio between the price at time $t$ of the $\complement_{d}$ (domestic) tradable asset $x_{f d}(t) A_{f}(t, \mathbf{S})$ and the numeraire $A_{d}(t, \mathbf{S})$, it must evolve according to a (driftless) martingale process under the associated discounting (domestic) swap measure $Q_{d}^{\mathbf{S}}$,

$$
\frac{d Y_{f d}(t, \mathbf{S})}{Y_{f d}(t, \mathbf{S})}=\nu_{Y}(t, \mathbf{S}) d W_{Y}^{\mathbf{S}}(t), \quad t \leq T_{0},
$$

where $v_{Y}(t, \mathbf{S})$ is the volatility (positive deterministic function of time) of the process and $W_{Y}^{\mathbf{S}}$ is a brownian motion under $Q_{d}^{\mathbf{S}}$ such that

$$
d W_{f}^{\mathbf{T}, \mathbf{S}}(t) d W_{Y}^{\mathbf{S}}(t)=\rho_{f Y}(t, \mathbf{T}, \mathbf{S}) d t .
$$


Now, applying again the change-of-numeraire technique of sec. 5.1, we obtain that the dynamic of the swap rate $S_{f}(t, \mathbf{T}, \mathbf{S})$ under the discounting (domestic) swap measure $Q_{d}^{\mathbf{S}}$ acquires a non-zero drift

$$
\begin{gathered}
\frac{d S_{f}(t, \mathbf{T}, \mathbf{S})}{S_{f}(t, \mathbf{T}, \mathbf{S})}=\lambda_{f}(t, \mathbf{T}, \mathbf{S}) d t+\nu_{f}(t, \mathbf{T}, \mathbf{S}) d W_{f}^{\mathbf{T}, \mathbf{S}}(t), \quad t \leq T_{0}, \\
\lambda_{f}(t, \mathbf{T}, \mathbf{S})=-\nu_{f}(t, \mathbf{T}, \mathbf{S}) \nu_{Y}(t, \mathbf{S}) \rho_{f Y}(t, \mathbf{T}, \mathbf{S})
\end{gathered}
$$

and that $S_{f}(t, \mathbf{T}, \mathbf{S})$ is lognormally distributed under $Q_{d}^{\mathbf{S}}$ with mean and variance given by

$$
\begin{aligned}
\mathbb{E}_{t}^{Q_{d}^{\mathbf{S}}}\left[\ln \frac{S_{f}\left(T_{0}, \mathbf{T}, \mathbf{S}\right)}{S_{f}(t, \mathbf{T}, \mathbf{S})}\right] & =\int_{t}^{T_{0}}\left[\lambda_{f}(u, \mathbf{T}, \mathbf{S})-\frac{1}{2} \nu_{f}^{2}(u, \mathbf{T}, \mathbf{S})\right] d u, \\
\operatorname{Var}_{t}^{Q_{d}^{\mathbf{S}}}\left[\ln \frac{S_{f}\left(T_{0}, \mathbf{T}, \mathbf{S}\right)}{S_{f}(t, \mathbf{T}, \mathbf{S})}\right] & =\int_{t f}^{T_{0}} \nu_{f}^{2}(u, \mathbf{T}, \mathbf{S}) d u .
\end{aligned}
$$

We thus obtain the following expressions, for $t_{0} \leq t<T_{0}$,

$$
\begin{aligned}
\mathbb{E}_{t}^{Q_{d}^{\mathbf{S}}}\left[S_{f}\left(T_{0}, \mathbf{T}, \mathbf{S}\right)\right] & =S_{f}(t, \mathbf{T}, \mathbf{S}) Q A_{f d}\left(t, \mathbf{T}, \mathbf{S}, \nu_{f}, \nu_{Y}, \rho_{f Y}\right), \\
Q A_{f d}\left(t, \mathbf{T}, \mathbf{S}, \nu_{f}, \nu_{Y}, \rho_{f Y}\right) & =\exp \int_{t}^{T_{0}} \lambda_{f}(u, \mathbf{T}, \mathbf{S}) d u \\
& =\exp \left[-\int_{t}^{T_{0}} \nu_{f}(u, \mathbf{T}, \mathbf{S}) \nu_{Y}(u, \mathbf{S}) \rho_{f Y}(u, \mathbf{T}, \mathbf{S}) d u\right]
\end{aligned}
$$

The same considerations as in sec. 5.1 apply. In particular, we observe that the adjustment in eqs. 55, 57 naturally follows from a change between the probability measures $Q_{f}^{\mathbf{S}}$ and $Q_{d}^{\mathbf{S}}$, or numeraires $A_{f}(t, \mathbf{S})$ and $A_{d}(t, \mathbf{S})$, associated to the two yield curves, $\complement_{f}$ and $\complement_{d}$, respectively, once swap rates are considered. In the EUR market, the volatility $\nu_{f}(u, \mathbf{T}, \mathbf{S})$ in eq. 56 can be extracted from quoted swaptions on Euribor6M, while for other rate tenors and for $\nu_{Y}(u, \mathbf{S})$ and $\rho_{f Y}(u, \mathbf{T}, \mathbf{S})$ one must resort to historical estimates.

An additive quanto adjustment can also be defined as before

$$
\begin{gathered}
\mathbb{E}_{t}^{Q_{d}^{\mathbf{S}}}\left[S_{f}\left(T_{0}, \mathbf{T}, \mathbf{S}\right)\right]=S_{f}(t, \mathbf{T}, \mathbf{S})+Q A_{f d}^{\prime}\left(t, \mathbf{T}, \mathbf{S}, \nu_{f}, \nu_{Y}, \rho_{f Y}\right), \\
Q A_{f d}^{\prime}\left(t, \mathbf{T}, \mathbf{S}, \nu_{f}, \nu_{Y}, \rho_{f Y}\right)=S_{f}(t, \mathbf{T}, \mathbf{S})\left[Q A_{f d}\left(t, \mathbf{T}, \mathbf{S}, \nu_{f}, \nu_{Y}, \rho_{f Y}\right)-1\right] .
\end{gathered}
$$

\section{Double-Curve Pricing \& Hedging Interest Rate Deriva- tives}

\subsection{Pricing}

The results of sec. 5 above allows us to derive no arbitrage, double-curve-single-currency pricing formulas for interest rate derivatives. The recipes are, basically, eqs. 37-38 or $55,56$.

The simplest interest rate derivative is a floating zero coupon bond paying at time $T$ a single cash flow depending on a single spot rate (e.g. the Xibor) fixed at time $t<T$,

$$
\mathbf{Z C B}(T ; T, N)=N \tau_{f}(t, T) L_{f}(t, T) .
$$


Being

$$
L_{f}(t, T)=\frac{1-P_{f}(t, T)}{\tau_{f}(t, T) P_{f}(t, T)}=F_{f}(t ; t, T),
$$

the price at time $t \leq T$ is given by

$$
\begin{aligned}
\mathbf{Z C B}(t ; T, N) & =N P_{d}(t, T) \tau_{f}(t, T) \mathbb{E}_{t}^{Q_{d}^{T}}\left[F_{f}(t ; t, T)\right] \\
& =N P_{d}(t, T) \tau_{f}(t, T) L_{f}(t, T) .
\end{aligned}
$$

Notice that the forward basis in eq. 61 disappears and we are left with the standard pricing formula, modified according to the double-curve framework.

Next we have the FRA, whose payoff is given in eq. [6 and whose price at time $t \leq T_{1}$ is given by

$$
\begin{array}{r}
\operatorname{FRA}\left(t ; T_{1}, T_{2}, K, N\right)=N P_{d}\left(t, T_{2}\right) \tau_{f}\left(T_{1}, T_{2}\right)\left\{\mathbb{E}_{t}^{Q_{d}^{T_{2}}}\left[F_{f}\left(T_{1} ; T_{1}, T_{2}\right)\right]-K\right\} \\
=N P_{d}\left(t, T_{2}\right) \tau_{f}\left(T_{1}, T_{2}\right)\left[F_{f}\left(t ; T_{1}, T_{2}\right) Q A_{f d}\left(t, T_{1}, \sigma_{f}, \sigma_{X}, \rho_{f X}\right)-K\right] .
\end{array}
$$

Notice that in eq. 62 for $K=0$ and $T_{1}=t$ we recover the zero coupon bond price in eq. 61.

For a (payer) floating vs fixed swap with payment dates vectors $\mathbf{T}, \mathbf{S}$ as in sec. 5.2 we have the price at time $t \leq T_{0}$

$$
\begin{aligned}
& \operatorname{Swap}(t ; \mathbf{T}, \mathbf{S}, \mathbf{K}, \mathbf{N}) \\
& =\sum_{i=1}^{n} N_{i} P_{d}\left(t, T_{i}\right) \tau_{f}\left(T_{i-1}, T_{i}\right) F_{f}\left(t ; T_{i-1}, T_{i}\right) Q A_{f d}\left(t, T_{i-1}, \sigma_{f, i}, \sigma_{X, i}, \rho_{f X, i}\right) \\
& -\sum_{j=1}^{m} N_{j} P_{d}\left(t, S_{j}\right) \tau_{d}\left(S_{j-1}, S_{j}\right) K_{j} .
\end{aligned}
$$

For constant nominal $N$ and fixed rate $K$ the fair (equilibrium) swap rate is given by

$$
S_{f}(t, \mathbf{T}, \mathbf{S})=\frac{\sum_{i=1}^{n} P_{d}\left(t, T_{i}\right) \tau_{f}\left(T_{i-1}, T_{i}\right) F_{f}\left(t ; T_{i-1}, T_{i}\right) Q A_{f d}\left(t, T_{i-1}, \sigma_{f, i}, \sigma_{X, i}, \rho_{f X, i}\right)}{A_{d}(t, \mathbf{S})}
$$

where

$$
A_{d}(t, \mathbf{S})=\sum_{j=1}^{m} P_{d}\left(t, S_{j}\right) \tau_{d}\left(S_{j-1}, S_{j}\right)
$$

is the annuity on curve $\complement_{d}$.

For caplet/floorlet options on a $T_{1}$-spot rate with payoff at maturity $T_{2}$ given by

$$
\operatorname{cf}\left(T_{2} ; T_{1}, T_{2}, K, \omega, N\right)=N \operatorname{Max}\left\{\omega\left[L_{f}\left(T_{1}, T_{2}\right)-K\right]\right\} \tau_{f}\left(T_{1}, T_{2}\right),
$$

the standard market-like pricing expression at time $t \leq T_{1} \leq T_{2}$ is modified as follows

$$
\begin{aligned}
& \mathbf{c f}\left(t ; T_{1}, T_{2}, K, \omega, N\right)=N \mathbb{E}_{t}^{Q_{d}^{T_{2}}}\left[\operatorname{Max}\left\{\omega\left[L_{f}\left(T_{1}, T_{2}\right)-K\right]\right\} \tau_{f}\left(T_{1}, T_{2}\right)\right] \\
& \quad=N P_{d}\left(t, T_{2}\right) \tau_{f}\left(T_{1}, T_{2}\right) B l\left[F_{f}\left(t ; T_{1}, T_{2}\right) Q A_{f d}\left(t, T_{1}, \sigma_{f}, \sigma_{X}, \rho_{f X}\right), K, \mu_{f}, \sigma_{f}, \omega\right],
\end{aligned}
$$


where $\omega=+/-1$ for caplets/floorlets, respectively, and

$$
\begin{gathered}
B l[F, K, \mu, \sigma, \omega]=\omega\left[F \Phi\left(\omega d^{+}\right)-K \Phi\left(\omega d^{-}\right)\right], \\
d^{ \pm}=\frac{\ln \frac{F}{K}+\mu(t, T) \pm \frac{1}{2} \sigma^{2}(t, T)}{\sigma(t, T)} \\
\mu(t, T)=\int_{t}^{T} \mu(u) d u, \quad \sigma^{2}(t, T)=\int_{t}^{T} \sigma^{2}(u) d u,
\end{gathered}
$$

is the standard Black-Scholes formula. Hence cap/floor options prices are given at $t \leq T_{0}$ by

$$
\begin{aligned}
& \mathbf{C F}(t ; \mathbf{T}, \mathbf{K}, \omega, \mathbf{N})=\sum_{i=1}^{n} \mathbf{c f}\left(T_{i} ; T_{i-1}, T_{i}, K_{i}, \omega_{i}, N_{i}\right) \\
& =\sum_{i=1}^{n} N_{i} P_{d}\left(t, T_{i}\right) \tau_{f}\left(T_{i-1}, T_{i}\right) \\
& \times B l\left[F_{f}\left(t ; T_{i-1}, T_{i}\right) Q A_{f d}\left(t, T_{i-1}, \sigma_{f, i}, \sigma_{X, i} \rho_{f X, i}\right), K_{i}, \mu_{f, i}, \sigma_{f, i}, \omega_{i}\right]
\end{aligned}
$$

Finally, for swaptions on a $T_{0}$-spot swap rate with payoff at maturity $T_{0}$ given by

$$
\text { Swaption }\left(T_{0} ; \mathbf{T}, \mathbf{S}, K, N\right)=N \operatorname{Max}\left[\omega\left(S_{f}\left(T_{0}, \mathbf{T}, \mathbf{S}\right)-K\right)\right] A_{d}\left(T_{0}, \mathbf{S}\right) \text {, }
$$

the standard market-like pricing expression at time $t \leq T_{0}$, using the discounting swap measure $Q_{d}^{\mathbf{S}}$ associated to the numeraire $A_{d}(t, \mathbf{S})$ on curve $\complement_{d}$, is modified as follows

$$
\begin{aligned}
\operatorname{Swaption}(t & ; \mathbf{T}, \mathbf{S}, K, N)=N A_{d}(t, \mathbf{S}) \mathbb{E}_{t}^{Q_{d}^{\mathbf{S}}}\left\{\operatorname{Max}\left[\omega\left(S_{f}\left(T_{0}, \mathbf{T}, \mathbf{S}\right)-K\right)\right]\right\} \\
& =N A_{d}(t, \mathbf{S}) B l\left[S_{f}(t, \mathbf{T}, \mathbf{S}) Q A_{f d}\left(t, \mathbf{T}, \mathbf{S}, \nu_{f}, \nu_{Y}, \rho_{f Y}\right), K, \lambda_{f}, \nu_{f}, \omega\right] .
\end{aligned}
$$

where we have used eq. 55 and the quanto adjustment term $Q A_{f d}\left(t, \mathbf{T}, \mathbf{S}, \nu_{f}, \nu_{Y}, \rho_{f Y}\right)$ is given by eq. 56 .

When two or more different underlying interest-rates are present, pricing expressions may become more involved. An example is the spread option, for which the reader can refer to, e.g., ch. 14.5.1 in ref. [BM06].

The calculations above show that also basic interest rate derivatives prices include a quanto adjustment and are thus volatility and correlation dependent. The volatilities and the correlation in eqs. 34 and 52 can be inferred from market data. In the EUR market the volatilities $\sigma_{f}$ and $\nu_{f}$ can be extracted from quoted caps/floors/swaptions on Euribor6M, while for $\sigma_{X}, \rho_{f X}$ and $\nu_{Y}, \rho_{f Y}$ one must resort to historical estimates. Conversely, given a forward basis term structure, such that in fig. 3, one could take $\sigma_{f}$ and $\nu_{f}$ from the market options, assume for simplicity $\rho_{f X} \simeq \rho_{f Y} \simeq 1$ (or any other functional form), and bootstrap out a term structure for the forward exchange rate volatilities $\sigma_{X}$ and $\nu_{Y}$. Notice that in this way one is also able to compare information about the internal dynamics of different market sub-areas.

In fig. [6] we show some numerical scenario for the quanto adjustment in eqs. [38, 40, We see that, for realistic values of volatilities and correlation, the magnitude of the additive adjustment may be non negligible, ranging from a few basis points up to over 10 basis 

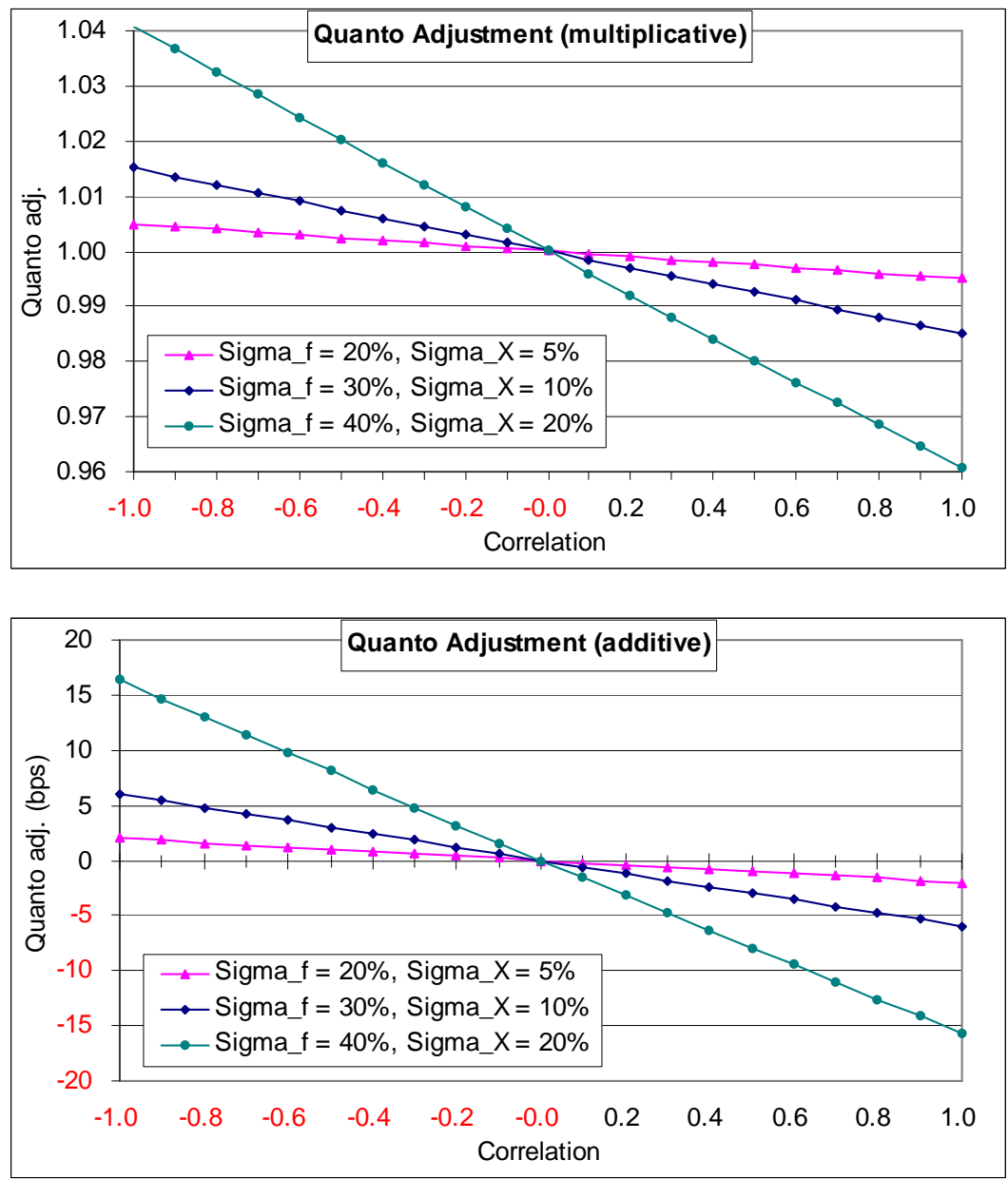

Figure 6: Numerical scenarios for the quanto adjustment. Upper panel: multiplicative (from eq. 38); lower panel: additive (from eq. 40). In each figure we show the quanto adjustment corresponding to three different combinations of (flat) volatility values as a function of the correlation. The time interval is fixed to $T_{1}-t=0.5$ and the forward rate entering eq. 40 to $4 \%$, a typical value in fig. 2. We see that, for realistic values of volatilities and correlation, the magnitude of the adjustment may be important.

points. Time intervals longer than the $6 \mathrm{M}$ period used in fig. 6 further increase the effect. Notice that positive correlation implies negative adjustment, thus lowering the forward rates that enters the pricing formulas above. Through historical estimation of parameters we obtain, using eq. 28 with the same yield curves as in fig. 3 and considering one year of backward data, forward exchange rate volatilities below 5\%-10\% and correlations within the range $[-0.6 ;+0.4]$.

Pricing interest rate derivatives without the quanto adjustment thus leaves, in principle, the door open to arbitrage opportunities. In practice the correction depends on financial variables presently not quoted on the market, making virtually impossible to set up arbitrage positions and lock today expected future positive gains. Obviously one may bet on his/her personal views of future realizations of volatilities and correlation. 


\subsection{Hedging}

Hedging within the multi-curve framework implies taking into account multiple bootstrapping and hedging instruments. We assume to have a portfolio $\Pi$ filled with a variety of interest rate derivatives with different underlying rate tenors. The first issue is how to calculate the delta sensitivity of $\Pi$. In principle, the answer is straightforward: having recognized interest-rates with different tenors as different underlyings, and having constructed $N_{C}$ multiple yield curves $\complement=\left\{\complement_{d}, \complement_{f}^{1}, \ldots, \complement_{f}^{N}\right\}$ using homogeneous market instruments, we must coherently calculate the sensitivity with respect to the corresponding market rates $\mathbf{r}^{B}=\left\{\mathbf{r}_{d}^{B}, \mathbf{r}_{f_{1}}^{B}, \ldots, \mathbf{r}_{f_{N}}^{B}\right\}$ of each bootstrapping instrument of each curve 11 ,

$$
\Delta^{B}\left(t, \mathbf{r}^{B}\right)=\sum_{i=1}^{N_{B}} \Delta_{i}^{B}\left(t, \mathbf{r}^{B}\right)=\sum_{k=1}^{N_{C}} \sum_{j=1}^{N_{k}^{r}} \frac{\partial \Pi\left(t, \mathbf{r}^{B}\right)}{\partial r_{j, k}^{B}}
$$

where $N_{B}$ is the total number of independent bootstrapping market rates, $N_{k}^{r}$ is the number of independent market rates in curve $\complement_{\mathbf{k}}, r_{j, k}^{B}$ is the $j$-th independent bootstrapping market rate of curve $\complement_{\mathbf{k}}$ and $\Pi\left(t, \mathbf{r}^{B}\right)$ is the price at time $t$ of the portfolio $\Pi$.

In practice this can be computationally cumbersome, given the high number of market instruments involved. Furthermore, second order delta sensitivities appear, due to the multiple curve bootstrapping described in sec. 3.2. In particular, the forwarding curves $\left\{\complement_{f}^{1}, \ldots, \complement_{f}^{N}\right\}$ depend directly on their corresponding bootstrapping market instruments $\left\{\mathbf{r}_{f_{1}}^{B}, \ldots, \mathbf{r}_{f_{N}}^{B}\right\}$ but also indirectly on the discounting curve $\complement_{d}$, as

$$
\begin{aligned}
\Delta^{B}\left(t, \mathbf{r}_{d}^{B}\right) & =\sum_{j=1}^{N_{d}^{r}} \sum_{\alpha=1}^{N_{d}^{z}} \frac{\partial \Pi\left(t, \mathbf{r}^{B}\right)}{\partial z_{d, \alpha}^{B}} \frac{\partial z_{d, \alpha}^{B}}{\partial r_{d, j}^{B}} \\
& +\sum_{j=1}^{N_{f}^{r}} \sum_{\alpha=1}^{N_{f}^{z}} \frac{\partial \Pi\left(t, \mathbf{r}^{B}\right)}{\partial z_{f, \alpha}^{B}}\left(\frac{\partial z_{f, \alpha}^{B}}{\partial r_{f, j}^{B}}+\frac{\partial z_{f, \alpha}^{B}}{\partial r_{d, j}^{B}}\right),
\end{aligned}
$$

where $\mathbf{z}^{B}=\left\{\mathbf{z}_{d}^{B}, \mathbf{z}_{f_{1}}^{B}, \ldots, \mathbf{z}_{f_{N}}^{B}\right\}$ is the vector of $\left\{N_{d}^{z}, N_{f_{1}}^{z}, \ldots, N_{f_{N}}^{z}\right\}$ zero rate pillars in the curves.

Once the delta sensitivity of the portfolio is known for each pillar of each relevant curve, the next issues of hedging are the choice of the set $\mathbf{H}$ of hedging instruments and the calculation of the corresponding hedge ratios $\mathbf{h}$. In principle, there are two alternatives: a) the set $\mathbf{H}$ of hedging instruments exactly overlaps the set $\mathbf{B}$ of bootstrapping instruments $(\mathbf{H} \equiv \mathbf{B})$; or, b) it is a subset restricted to the most liquid bootstrapping instruments $(\mathbf{H} \subset \mathbf{B})$. The first choice allows for a straightforward calculation of hedge ratios and representation of the delta risk distribution of the portfolio. But, in practice, people prefer to hedge using the most liquid instruments, both for better confidence in their market prices and for reducing the cost of hedging. Hence the second strategy generally prevails. In this case the calculation of hedge ratios requires a three-step procedure: first, the sensitivity $\boldsymbol{\Delta}^{B}=\left\{\Delta_{1}^{B}, \ldots, \Delta_{N_{B}}^{B}\right\}$ is calculated as in eq. 74 on the basis $\mathbf{B}$ of all bootstrapping instruments; second, $\Delta^{B}$ is projected onto the basis $\mathbf{H}$ of hedging instrument $\left\{\right.$, characterized by market rates $\mathbf{r}^{H}=\left\{r_{1}^{H}, \ldots, r_{N_{H}}^{H}\right\}$, thus obtaining the

\footnotetext{
${ }^{11}$ with the obvious caveat of avoiding double counting of those instruments eventually appearing in more than one curve (3M Futures for instance could appear both in $\complement_{d}$ and in $\complement_{f}^{3 M}$ curves).

${ }^{12}$ in practice $\boldsymbol{\Delta}^{H}$ is obtained by aggregating the components $\boldsymbol{\Delta}_{i}^{B}$ through appropriate mapping rules.
} 
components $\Delta^{H}=\left\{\Delta_{1}^{H}, \ldots, \Delta_{N_{H}}^{H}\right\}$ with the constrain

$$
\Delta^{B}=\sum_{i=1}^{N_{B}} \Delta_{i}^{B}=\sum_{j=1}^{N_{H}} \Delta_{j}^{H}=\Delta^{H} ;
$$

then, hedge ratios $\mathbf{h}=\left\{h_{1}, \ldots, h_{N_{H}}\right\}$ are calculated as

$$
h_{j}=\frac{\Delta_{j}^{H}}{\delta_{j}^{H}},
$$

where $\delta^{H}=\left\{\delta_{1}^{H}, \ldots, \delta_{N_{H}}^{H}\right\}$ is the delta sensitivity of the hedging instruments. The disadvantage of this second choice is, clearly, that some risk - the basis risk in particular is only partially hedged; hence, a particular care is required in the choice of the hedging instruments.

A final issue regards portfolio management. In principle one could keep all the interest rate derivatives together in a single portfolio, pricing each one with its appropriate forwarding curve, discounting all cash flows with the same discounting curve, and hedging using the preferred choice described above. An alternative is the segregation of homogeneous contracts (with the same underlying interest rate index) into dedicated sub-portfolios, each managed with its appropriate curves and hedging techniques. The (eventually) remaining non-homogeneous instruments (those not separable in pieces depending on a single underlying) can be redistributed in the portfolios above according to their prevailing underlying (if any), or put in other isolated portfolios, to be handled with special care. The main advantage of this second approach is to "clean up" the trading books, "cornering" the more complex deals in a controlled way, and to allow a clearer and self-consistent representation of the sensitivities to the different underlyings, and in particular of the basis risk of each sub-portfolio, thus allowing for a cleaner hedging.

\section{$7 \quad$ No Arbitrage and Counterparty Risk}

Both the forward basis and the quanto adjustment discussed in sections 4, 5 above find a simple financial explanation in terms of counterparty risk. From this point of view we may identify $P_{d}(t, T)$ with a default free zero coupon bond and $P_{f}(t, T)$ with a risky zero coupon bond with recovery rate $R_{f}$, emitted by a generic interbank counterparty subject to default risk. The associated risk free and risky Xibor rates, $L_{d}\left(T_{1}, T_{2}\right)$ and $L_{f}\left(T_{1}, T_{2}\right)$, respectively, are the underlyings of the corresponding derivatives, e.g. $\mathrm{FRA}_{d}$ and $\mathrm{FRA}_{f}$. Adapting the simple credit model proposed in ref. [Mer09] we may write, using our notation,

$$
\begin{aligned}
P_{f}(t, T) & =P_{d}(t, T) R\left(t ; t, T, R_{f}\right), \\
R\left(t ; T_{1}, T_{2}, R_{f}\right) & :=R_{f}+\left(1-R_{f}\right) \mathbb{E}_{t}^{Q_{d}}\left[q_{d}\left(T_{1}, T_{2}\right)\right],
\end{aligned}
$$

where $q_{d}(t, T)=\mathbb{E}_{t}^{Q_{d}}\left[1_{\tau(t)>T}\right]$ is the counterparty default probability after time $T$ expected at time $t$ under the risk neutral discounting measure $Q_{d}$. Using eqs. 778, 79 we 
may express the risky Xibor spot and forward rates as

$$
\begin{aligned}
L_{f}\left(T_{1}, T_{2}\right) & =\frac{1}{\tau_{f}\left(T_{1}, T_{2}\right)}\left[\frac{1}{P_{f}\left(T_{1}, T_{2}\right)}-1\right] \\
& =\frac{1}{\tau_{f}\left(T_{1}, T_{2}\right)}\left[\frac{1}{P_{d}\left(T_{1}, T_{2}\right)} \frac{1}{R\left(T_{1} ; T_{1}, T_{2}, R_{f}\right)}-1\right], \\
F_{f}\left(t ; T_{1}, T_{2}\right) & =\frac{1}{\tau_{f}\left(T_{1}, T_{2}\right)}\left[\frac{P_{f}\left(t, T_{1}\right)}{P_{f}\left(t, T_{2}\right)}-1\right] \\
& =\frac{1}{\tau_{f}\left(T_{1}, T_{2}\right)}\left[\frac{P_{d}\left(t, T_{1}\right)}{P_{d}\left(t, T_{2}\right)} \frac{R\left(t ; t, T_{1}, R_{f}\right)}{R\left(t ; t, T_{2}, R_{f}\right)}-1\right],
\end{aligned}
$$

and the risky $\mathrm{FRA}_{f}$ price at time $t$ as 13

$$
\operatorname{FRA}_{f}\left(t ; T_{1}, T_{2}, K\right)=\frac{P_{d}\left(t, T_{1}\right)}{R\left(t ; T_{1}, T_{2}, R_{f}\right)}-P_{d}\left(t, T_{2}\right)\left[1+K \tau_{f}\left(T_{1}, T_{2}\right)\right]
$$

Introducing eq. 81 in eqs. 20 and 22 we obtain the following expressions for the forward basis

$$
\begin{aligned}
B A_{f d}\left(t ; T_{1}, T_{2}\right) & =\frac{P_{d}\left(t, T_{1}\right) R\left(t ; t, T_{1}, R_{f}\right)-P_{d}\left(t, T_{2}\right) R\left(t ; t, T_{2}, R_{f}\right)}{\left[P_{d}\left(t, T_{1}\right)-P_{d}\left(t, T_{2}\right)\right] R\left(t ; t, T_{2}, R_{f}\right)} \\
B A_{f d}^{\prime}\left(t ; T_{1}, T_{2}\right) & =\frac{1}{\tau_{d}\left(T_{1}, T_{2}\right)} \frac{P_{d}\left(t, T_{1}\right)}{P_{d}\left(t, T_{2}\right)}\left[\frac{R\left(t ; t, T_{1}, R_{f}\right)}{R\left(t ; t, T_{2}, R_{f}\right)}-1\right] .
\end{aligned}
$$

From the FRA pricing expression eq. 62 we may also obtain an expression for the FRA quanto adjustment

$$
\begin{aligned}
Q A_{f d}\left(t ; T_{1}, T_{2}\right) & =\frac{P_{d}\left(t, T_{1}\right) \frac{1}{R\left(t ; T_{1}, T_{2}, R_{f}\right)}-P_{d}\left(t, T_{2}\right)}{P_{d}\left(t, T_{1}\right) \frac{R\left(t ; t, T_{1}, R_{f}\right)}{R\left(t ; t, T_{2}, R_{f}\right)}-P_{d}\left(t, T_{2}\right)}, \\
Q A_{f d}^{\prime}\left(t ; T_{1}, T_{2}\right) & =\frac{1}{\tau_{f}\left(T_{1}, T_{2}\right)} \frac{P_{d}\left(t, T_{1}\right)}{P_{d}\left(t, T_{2}\right)}\left[\frac{1}{R\left(t ; T_{1}, T_{2}, R_{f}\right)}-\frac{R\left(t ; t, T_{1}, R_{f}\right)}{R\left(t ; t, T_{2}, R_{f}\right)}\right] .
\end{aligned}
$$

Thus the forward basis and the quanto adjustment can be expressed, under simple credit assumptions, in terms of risk free zero coupon bonds, survival probability and recovery rate. A more complex credit model, as e.g. in ref. [Mor09], would also be able to express the spot exchange rate in eq. 28 in terms of credit variables. Notice that the single-curve case $\mathcal{C}_{d}=\mathcal{C}_{f}$ is recovered for vanishing default risk (full recovery).

\section{Conclusions}

We have discussed how the liquidity crisis and the resulting changes in the market quotations, in particular the very high basis swap spreads, have forced the market practice to evolve the standard procedure adopted for pricing and hedging single-currency interest rate derivatives. The new double-curve framework involves the bootstrapping of multiple

\footnotetext{
${ }^{13}$ in particular, contrary to ref. [Mer09], we use here the FRA definition of eq. 8, leading to eq. 82
} 
yield curves using separated sets of vanilla interest rate instruments homogeneous in the underlying rate (typically with $1 \mathrm{M}, 3 \mathrm{M}, 6 \mathrm{M}, 12 \mathrm{M}$ tenors). Prices, sensitivities and hedge ratios of interest rate derivatives on a given underlying rate tenor are calculated using the corresponding forward curve with the same tenor, plus a second distinct curve for discount factors.

We have shown that the old, well-known, standard single-curve no arbitrage relations are no longer valid and can be recovered with the introduction of a forward basis, for which simple statical expressions are given in eqs. 20,22 in terms of discount factors from the two curves. Our numerical results have shown that the forward basis curves, in particular in a realistic stressed market situation, may display an oscillating term structure, not present in the smooth and monotonic basis swaps market quotes and more complex than that of the discount and forward curves. Such richer micro-term structure is caused by amplification effects of small local differences between the discount and forwarding curves and constitutes both a very sensitive test of the quality of the bootstrapping procedure (interpolation in particular), and an indicator of the tiny, but observable, differences between different interest rate market areas. Both of these causes may have appreciable effects on the price of interest rate instruments, in particular when one switches from the single-curve towards the double-curve framework.

Recurring to the foreign-currency analogy we have also been able to recompute the no arbitrage double-curve-single-currency market-like pricing formulas for basic interest rate derivatives, zero coupon bonds, FRA, swaps caps/floors and swaptions in particular. Such prices depend on forward or swap rates on curve $\complement_{f}$ corrected with the well-known quanto adjustment typical of cross-currency derivatives, naturally arising from the change between the numeraires, or probability measures, naturally associated to the two yield curves. The quanto adjustment depends on the volatility $\sigma_{f}$ of the forward rates $F_{f}$ on $\complement_{f}$, of the volatility $\sigma_{X}$ of the forward exchange rate $X_{f d}$ between $\complement_{f}$ and $\complement_{d}$, and of the correlation $\rho_{f X}$ between $F_{f}$ and $X_{f d}$. In particular, a non-trivial adjustment is obtained if and only if the forward exchange rates $X_{f d}$ are stochastic $\left(\sigma_{X} \neq 0\right)$ and correlated to the forward rate $F_{f}\left(\rho_{f X} \neq 0\right)$. Analogous considerations hold for the swap rate quanto adjustment. Numerical scenarios show that the quanto adjustment can be important, depending on volatilities and correlation. Unadjusted interest rate derivatives' prices are thus, in principle, not arbitrage free, but, in practice, at the moment the market does not trade enough instruments to set up arbitrage positions.

Finally, both the forward basis and the quanto adjustment find a natural financial explanation in terms of counterparty risk within a simple credit model including a default free and a risky zero coupon bond.

Besides the lack of information about volatility and correlation, the present framework has the advantage of introducing a minimal set of parameters with a transparent financial interpretation and leading to familiar pricing formulas, thus constituting a simple and easy-to-use tool for practitioners and traders to promptly intercept possible market evolutions. 


\section{References}

[AB09] Ferdinando M. Ametrano and Marco Bianchetti. Smooth yield curves bootstrapping for forward libor rate estimation and pricing interest rate derivatives. In Fabio Mercurio, editor, Modelling Interest Rates: Latest Advances for Derivatives Pricing. Risk Books, 2009.

[And07] Leif B.G. Andersen. Discount curve construction with tension splines. Review of Derivatives Research, 10(3):227-267, December 2007.

[BM06] Damiano Brigo and Fabio Mercurio. Interest-Rate Models - Theory and Practice. Springer, 2 edition, 2006.

[BS05] Wolfram Boenkost and Wolfgang M. Schmidt. Cross currency swap valuation. Working paper, HfB-Business School of Finance \& Management, May 2005.

[FZW95] Emmanuel Fruchard, Chaker Zammouri, and Edward Willems. Basis for change. Risk Magazine, 8(10):70-75, 1995.

[GKR95] Henliette Geman, Nicole El Karoui, and Jean-Charles Rochet. Changes of numeraire, changes of probability measure and option pricing. Journal of Applied Probability, 32(2):443-458, 1995.

[GS009] The future of interest rates swaps. presentation, Goldman Sachs, February 2009.

[Hen07] Marc P. A. Henrard. The irony in the derivatives discounting. Wilmott Magazine, Jul/Aug 2007.

[Hen09] Marc P. A. Henrard. The irony in the derivatives discounting part ii: The crisis. SSRN Working paper, Jul 2009.

[HW06] Patrick. S. Hagan and Graeme West. Interpolation methods for curve construction. Applied Mathematical Finance, 13(2):89-129, June 2006.

[HW08] Patrick. S. Hagan and Graeme West. Methods for constructing a yield curve. Wilmott Magazine, pages 70-81, 2008.

[Jam89] Farshid Jamshidian. An exact bond option pricing formula. Journal of Finance, 44:205-209, 1989.

[KTW08] M. Kijima, K. Tanaka, and T. Wong. A multi-quality model of interest rates. Quantitative Finance, 2008.

[Mad08] Peter Madigan. Libor under attack. Risk Magazine, June 2008.

[Mer09] Fabio Mercurio. Post credit crunch interest rates: Formulas and market models. SSRN Working paper, 2009.

[Mor08] Massimo Morini. Credit modelling after the subprime crisis. Marcus Evans course, 2008. 
[Mor09] Massimo Morini. Solving the puzzle in the interest rate market. SSRN Working paper, 2009.

[Qua09] QuantLib, the free/open-source object oriented c++ financial library. Release 0.9.9-2009, 2009.

[Ron00] Uri Ron. A practical guide to swap curve construction. Working Paper 2000-17, Bank of Canada, August 2000.

[TP03] Bruce Tuckman and Pedro Porfirio. Interest rate parity, money market basis swaps, and cross-currency basis swaps. Fixed income liquid markets research, Lehman Brothers, June 2003. 